\title{
Can Förster Theory Describe Stereoselective Energy Transfer Dynamics in a Protein-Ligand Complex?
}

\author{
Silvana Pinheiro ${ }^{1}$ and Carles Curutchet ${ }^{1, *}$ \\ ${ }^{1}$ Departament de Farmàcia i Tecnologia Farmacèutica i Fisicoquímica and Institut de \\ Biomedicina (IBUB), Facultat de Farmàcia i Ciències de l'Alimentació, Universitat de \\ Barcelona, Barcelona Spain \\ *Corresponding author: CC (carles.curutchet@ub.edu)
}

\begin{abstract}
Förster resonance energy transfer (FRET) reactions involving ligands and aromatic amino acids can substantially impact the fluorescence properties of a protein-ligand complex, an impact intimately related to the corresponding binding mode. Structural characterization of such binding events in terms of intermolecular distances can be done through the well-known $\mathrm{R}^{-6}$ distance-dependent Förster rate expression. However, such interpretation suffers from uncertainties underlying Förster theory in the description of the electronic coupling that promotes FRET, mostly related to the dipole-dipole orientation factor, dielectric screening effects and deviations from the ideal dipole approximation. Here, we investigate how Förster approximations impact the prediction of energy transfer dynamics in the complex between flurbiprofen and human serum albumin (HSA), as well as a model flurbiprofen-Trp dyad, in which recent observations of enantioselective fluorescence quenching has been ascribed to energy transfer from flurbiprofen to Trp. To this aim, we combine classical molecular dynamics simulations with polarizable quantum mechanics/molecular mechanics $(\mathrm{QM} / \mathrm{MM})$ calculations that allow overcoming Förster approximations. On the basis of our results, we discuss the potential of structure-based simulations in the characterization of drug-binding events through fluorescence techniques. Overall, we find an excellent agreement among theory and experiment both in terms of enantioselectivity and FRET times, thus strongly supporting the reliability of the binding modes proposed for the (S)- and (R)enantiomers of flurbiprofen. In particular, we show that the dynamic quenching arises from a small fraction of drug bound to the secondary site of HSA at the interface between subdomains IIA and IIB, whereas the enantioselectivity arises from the larger flexibility of the (S)-flurbiprofen enantiomer in the binding pocket.
\end{abstract}




\section{INTRODUCTION}

The fluorescence of proteins is exquisitely dependent on structural changes, a property that has led to a plethora of techniques aimed at assessing protein structure and dynamics. ${ }^{1-10}$ Such fluorescence generally arises from Trp residues in the structure, whose emission wavelength, quantum yield and lifetimes are exquisitely sensitive to the surrounding environment. ${ }^{2,8,11,12}$ Indeed, the protein scaffold not only tunes the emission wavelength of Trp by electrostatic interactions, but also mediates proton, electron and energy transfer reactions that can efficiently quench Trp emission, thus modifying its quantum yield and lifetime. ${ }^{13}$ In a protein-ligand complex, the bound ligand can further modify the fluorescence properties of the protein by participating in Förster resonance energy transfer (FRET) reactions with Trp, an impact that is intimately related to the particular binding mode adopted by the ligand. Conversely, the ligand fluorescence can also be drastically modulated upon protein binding. ${ }^{14}$

Structural characterization of ligand binding events from FRET data based on the popular Förster expression, which predicts a $\mathrm{R}^{-6}$ distance decay of the transfer rate with donor-acceptor separation, suffers however from uncertainties related to Förster description of the donor-acceptor electronic coupling. Such limitations are related to the orientation factor, dielectric screening effects exerted by the surrounding environment, and potential deviations from the ideal dipole approximation. ${ }^{15}$ For example, because a ligand has a restricted rotational freedom in the protein binding site, an isotropic approximation for the dipole-dipole orientation factor, $\left\langle\kappa^{2}\right\rangle=2 / 3$, will likely break down. ${ }^{16}$ In addition, if the ligand is close to the Trp residue, the coupling can significantly deviate from the ideal dipole description, specially if the rotational freedom of the ligand is restricted. ${ }^{17}$ Finally, dielectric screening effects strongly depend on the nature of the amino acids that surround the chromophores involved in a FRET event. ${ }^{18}$ The potential of FRET experiments to characterize ligand binding events - its exquisite sensitivity to subtle structural changes in the underlying binding mode - is thus underexploited under a simple Förster-type interpretation in terms of intermolecular distances.

Different research groups have used molecular dynamics (MD) simulations in order to characterize FRET data in terms of structure. ${ }^{16,19-40}$ Krueger and co-workers, for example, have shown that $\kappa$ and $\mathrm{R}$ can be strongly correlated for a pair of fluorescent probes attached to lysozyme, thus modifying by $60 \%$ the predicted rates compared to the independent $\kappa$ and R assumption common in FRET studies. ${ }^{16}$ On the 
other hand, by combining MD and polarizable quantum mechanics/molecular mechanics (QM/MM) calculations, we have recently examined the impact of dielectric screening in several photosynthetic pigment-protein complexes. Our results showed that the heterogeneous nature of the environment can modulate by a factor up to $\sim 4$ FRET rates with respect to those predicted for a homogeneous medium, as assumed in Förster theory. ${ }^{18,41}$

In this study, we apply the MD-QM/MMpol strategy we have developed to describe energy migration in complex environments ${ }^{42}$ to examine the validity of Förster assumptions in FRET studies of ligand binding. In particular, we investigate the enantioselective fluorescence dynamic quenching recently observed by Vayá and coworkers in the complex between human serum albumin (HSA) and the (S)- and (R)enantiomers of flurbiprofen (FBP), which have been ascribed to excitation transfer from $1 F B *^{*}$ to the Trp214 residue in HSA. ${ }^{14}$ Characterizing the binding of drugs to HSA is important to understand their pharmacodynamic and pharmacokinetic profile, given that HSA is the major transport plasma protein. ${ }^{43,44}$ In this case, we study the binding of flurbiprofen, a chiral nonsteroidal anti-inflammatory drug (NSAID), in which the (S)enantiomer shows most of the anti-inflammatory activity, although both enantiomers posses analgesic activity. In addition, flurbiprofen exhibits stereoselectivity in its pharmacokinetics, ${ }^{45}$ and stereoselective binding to HSA has been shown to occur. ${ }^{46,47}$ It is known that flurbiprofen binds preferentially to site II (benzodiazepine binding site) of HSA but also binds with remarkable affinity to another site. ${ }^{46,48,49}$ Often this secondary binding site has been identified with well-known site I (warfarin binding site) of HSA. However, the crystal structure of ibuprofen bound to HSA, ${ }^{50}$ a drug with a rather similar structure and binding characteristics, ${ }^{48}$ indicates that the secondary site is not site I. Rather, it is a novel site located between subdomains IIA and IIB. This finding explains why there is only a partial quenching of HSA phosphorescence in the presence of FBP ${ }^{47}$ as FBP in that secondary site is not in direct contact with $\operatorname{Trp} 214$ as would be in site I, but at a distance $\sim 10 \AA .^{50}$

In addition to the FBP/HSA complex, we also examine covalently linked dyads formed by (S)- and (R)-enantiomers of FBP and (S)-tryptophan methyl ester (TrpMe), in which the enantioselectivity is reversed. ${ }^{14}$ For the FBP/HSA complexes, we explore the flexibility and binding modes of the enantiomers using classical MD simulations, then we estimate the FRET properties of the system from polarizable QM/MM excited state calculations performed along the MD trajectories. ${ }^{51}$ For the model dyads, we find 
that the classical force field is unable to describe their conformational properties in acetonitrile, so we determine the main conformers and evaluate the EET properties of the dyad based on static QM calculations coupled to a continuum solvation model. Interestingly, we find that the enantioselectivity in the model dyad arises from an almost orthogonal arrangement of the transition dipole moments of FBP and Trp in the (S,S)dyad, which explains the 2-3 times slower dynamic quenching observed. On the other hand, we find that the dynamic quenching in the FBP/HSA complex arises from the fraction of FBP bound to the secondary site of HSA at the interface between subdomains IIA and IIB, rather than the main population bound at site II. In this case, the enantioselective quenching arises from the increased flexibility of the (S)-FBP diastereomer compared to the (R)-FBP one, which induces large fluctuations in electronic couplings, in line with the faster reorientational time derived from fluorescence anisotropy for that enantiomer. ${ }^{14}$ In both model dyad and FBP/complexes, we find that a precise determination of the transition dipole orientation of $\operatorname{Trp} L_{a}$ state through time-dependent density functional theory (TD-DFT), as well as account for higher-order multipolar effects beyond the dipole approximation based on 3D transition densities, is necessary in order to capture the subtle changes in FRET rates observed.

The present contribution is organized as follows. First, we describe the methods used in this work. Then, we discuss the results for the model dyads followed by those obtained for the FBP/HSA complexes. Finally, we close with our conclusions and future prospects.

\section{METHODS}

\section{Förster energy transfer theory}

The interpretation of FRET studies is typically performed based on Förster theory, introduced more than 60 years ago, ${ }^{52,53}$ which describes the rate of non-radiative electronic excitation energy transfer from a donor (D) molecule to a weakly-coupled acceptor (A):

$$
k_{F R E T}=\frac{2 \pi}{\hbar} V^{2} J
$$

where $V$ describes the donor-acceptor electronic coupling, and $J$ the overlap factor between donor emission $f_{D}(\epsilon)$ and acceptor absorption lineshapes $a_{A}(\epsilon)$, both normalized to unit area on an energy scale:

$$
J=\int_{0}^{\infty} f_{D}(\epsilon) a_{A}(\epsilon) d \epsilon
$$


The distance and orientation dependence of the rate is mainly encapsulated in the $V^{2}$ term, which is approximated as:

$$
\begin{aligned}
& V \approx s V_{\text {dip-dip }} \\
& s=\frac{1}{n^{2}} \\
& V_{\text {dip-dip }}=\frac{\kappa \mu_{D}^{T} \mu_{A}^{T}}{R^{3}}
\end{aligned}
$$

where the orientation factor is given by

$$
\kappa=\hat{\mu}_{D}^{T} \cdot \hat{\mu}_{A}^{T}-3\left(\hat{\mu}_{D}^{T} \cdot \hat{R}\right)\left(\hat{\mu}_{A}^{T} \cdot \hat{R}\right)
$$

and $n$ indicates the refractive index of the medium, $\mu_{D}^{T} / \mu_{A}^{T}$ the $\mathrm{D} / \mathrm{A}$ transition dipole moments and $\mathrm{R}$ the intermolecular center-to-center separation $\left(\hat{\mu}_{D}^{T}, \hat{\mu}_{A}^{T}\right.$ and $\hat{R}$ are the corresponding unit vectors).

The derivation of Förster theory relies on the weak coupling approximation, according to which the electronic coupling is small compared to the reorganization energy of the chromophores. Although there's a vivid debate on theories valid for other coupling regimes, ${ }^{54}$ in particular in studies of light harvesting in photosynthesis, typically FRET studies are performed in conditions of weak coupling. In this cases, the most problematic assumptions related to Förster theory ${ }^{15}$ are the ideal dipole approximation used for the coupling in Eq. 5, as well as the rather crude dielectric screening factor based on the refractive index of the medium in Eq. 4, which does not depend on the local environment or the relative D/A arrangement. Moreover, the impossibility to determine the relative orientation of $\mathrm{D} / \mathrm{A}$ molecules often leads to the adoption of an isotropic average for the orientation factor, $\left\langle\kappa^{2}\right\rangle=2 / 3$.

\section{Polarizable QM/MM and QM/PCM models for energy transfer}

In order to overcome the limitations of Förster dielectric screening and dipoledipole approximations discussed above, we use two multiscale approaches we have developed to study energy transfer in condensed phases. These methods combine a quantum mechanical (QM) description of the chromophores (in this case FBP and Trp) with either an classical polarizable molecular mechanics (MMPol) description of the surrounding environment based on point charges and induced dipoles ${ }^{51}$ or a continuum solvation description based on the Polarizable Continuum Model (PCM). ${ }^{55,56}$ Both approaches take into account mutual polarization effects among the QM and MM regions during the self-consistent-field process and the excited-state environment response. The electronic couplings are then estimated from the transition densities 
derived from the $\mathrm{QM} / \mathrm{MMPol}$ or $\mathrm{QM} / \mathrm{PCM}$ excited state calculations, and dielectric screening effects are naturally incorporated by explicitly computing an environmentmediated term to the Coulomb coupling among D and A:

$$
V=V_{\text {coul }}+V_{\text {env }}
$$

The Coulomb and the environment-mediated terms in the QM/MMPol and QM/PCM formulations are given by

$$
\begin{gathered}
V_{\text {coul }}=\int d \boldsymbol{r} \int d \boldsymbol{r}^{\prime} \rho_{A}^{T *}(\boldsymbol{r}) \frac{1}{\left|\boldsymbol{r}^{\prime}-\boldsymbol{r}\right|} \rho_{D}^{T}(\boldsymbol{r}) \\
V_{M M P o l}=-\sum_{k} \int d \boldsymbol{r} \rho_{A}^{T *}(\boldsymbol{r}) \frac{\left(\boldsymbol{r}_{\boldsymbol{k}}-\boldsymbol{r}\right)}{\left|\boldsymbol{r}_{\boldsymbol{k}}-\boldsymbol{r}\right|^{3}} \boldsymbol{\mu}_{k}^{M M P o l}\left(\rho_{D}^{T}\right) \\
V_{P C M}=\sum_{l} \int d \boldsymbol{r} \rho_{A}^{T *}(\boldsymbol{r}) \frac{1}{\left|\boldsymbol{s}_{\boldsymbol{l}}-\boldsymbol{r}\right|} \boldsymbol{q}_{l}^{P C M}\left(\rho_{D}^{T}\right)
\end{gathered}
$$

where $\rho_{D / A}^{T}$ indicates the transition densities of the interacting pigments, and $\boldsymbol{\mu}_{k}^{M M P o l}$ and $\boldsymbol{q}_{l}^{P C M}$ the MMPol induced dipoles and PCM apparent surface charges describing the polarization response of the environment to a given transition density. The integrals in Eqs. 8-10 are solved using the efficient DFT grid-based integration methods implemented in the Gaussian code. ${ }^{42,55}$ The $V_{\text {coul }}$ term describes the Coulomb interaction between the transition densities, thus representing an extension of Förster dipole-dipole coupling when higher-multipolar contributions are included, i.e. when the shape of the interacting molecules is fully taken into account. Both PCM and MMPol terms depend on distance and mutual orientation of the chromophores, and typically counteract the Coulomb term thus leading to an overall screening of the interactions. One can thus define an effective screening factor s, that can be directly compared to Förster $\frac{1}{n^{2}}$ term in Eq. 4 :

$$
S=\frac{\left(V_{c o u l}+V_{e n v}\right)}{V_{c o u l}}
$$

\section{Molecular dynamics simulations}

We performed molecular dynamics simulations of the (S)- and (R)-FBP/HSA complexes, as well as the (S,S)- and (R,S)-FBP-TrpMe dyads. The (S,S)- and (R,S)FBP-TrpMe dyads were solvated in a $\mathrm{CH}_{3} \mathrm{CN}$ truncated octahedron box (buffer zone of $25 \AA$ ) using the Leap tool of the Amber 12 software. ${ }^{57}$ The FBP/HSA systems, on the other hand, were initially prepared from the crystal structure of HSA complexed with ibuprofen (PDB 2BXG), solved at a $2.7 \AA$ resolution, ${ }^{50}$ where the structures of the (S)and (R)-enantiomers of anionic FBP were aligned to the structure of ibuprofen. In 
particular, ibuprofen is bound to the well-known site II of HSA, but also to a secondary site of HSA located between subdomains IIA and IIB. ${ }^{50}$ We align FBP enantiomers in order to place them at this secondary site, as an energy transfer in the subnanosecond timescale is only compatible with that binding mode, populated by $30-40 \%$ of FBP enantiomers in the experimental conditions we aim at simulating, ${ }^{14,46}$ as will be discussed in the results section. We assumed a standard protonation state for all residues except Glu244, which was in the neutral state, as indicated by $\mathrm{pKa}$ estimates at neutral $\mathrm{pH}$ computed using the PROPKA3 server. ${ }^{58}$ The system was then solvated in a TIP3P ${ }^{59}$ water truncated octahedron box (buffer zone of $20 \AA$ ). We used the force field for $\mathrm{CH}_{3} \mathrm{CN}$ derived by Grabuleda et al. ${ }^{60}$ and the Amber ff $12 \mathrm{SB}$ force field for the protein, ${ }^{61,62}$ whereas the parameters for FBP and the FBP-TrpMe dyad were derived based on the Amber ff12SB force field ${ }^{61,62}$ using the Antechamber module of Amber 12. The torsional parameters describing the rotation of the bond connecting the FBP phenyl units was explicitly parametrized by comparing fully relaxed torsion profiles computed at $5^{\circ}$ intervals, in which the dihedral angle was frozen, obtained minimizing the FBP structure at the B3LYP-D3/6-31++G(d,p) level of theory (adopting D3 Grimme's dispersion correction ${ }^{63}$ ) and using the classical force field. The atomic charges for the FBP and the FBP-TrpMe enantiomers were computed following the RESP approach ${ }^{64}$ based on the HF/6-31G(d) electrostatic potential computed on a B3LYP/6-31++G(d,p) (dyads) and MP2/6-311++G(d,p) (FBP) optimized geometries. The FBP and FBP-TrpMe force field parameters are reported in the Supporting Information.

The (S,S)- and (R,S)-FBP-TrpMe dyads and the (S)- and (R)-FBP/HSA systems were first energy-minimized for 1000 steps (only solvent and ions) and then for 2500 steps allowing the complete systems to relax. Then, the systems were gradually thermalized by running five 100-ps simulations at constant volume to increase the temperature from 0 to $298 \mathrm{~K}$, and subsequently simulated in the isothermal-isobaric ensemble (1 atm and $298 \mathrm{~K}$ ) for a total of $100 \mathrm{~ns}$. For the (S)- and (R)-FBP/HSA systems, the equilibration plus production runs starting from the energy-minimized structures was repeated for a total of 5 replicas. All simulations were done using the Amber12 $\operatorname{code}^{57}$ with periodic boundary conditions, the Particle Mesh Ewald approach to account for long-range electrostatics, a non-bonded cutoff of $8 \AA$, a 2 fs integration time step, the SHAKE algorithm to restrain bonds involving hydrogen and the Langevin dynamics method to control the temperature (collision frequency $1 \mathrm{ps}^{-1}$ ). In constant 
pressure simulations, we used the isotropic position scaling algorithm (pressure relaxation time 2 ps). From the last $50 \mathrm{~ns}$ of each replica, a total of 100 snapshots were extracted each $0.5 \mathrm{~ns}$ to be used in the QM/classical calculations.

\section{QM/MMPol and QM/PCM calculations}

QM geometry optimizations of the model dyad structures extracted from the MD simulations were done at the B3LYP/6-31G(d) level of theory adopting D3 Grimme's dispersion correction ${ }^{63}$ and the SMD solvation model ${ }^{65}$ in acetonitrile. We then performed a frequency analysis in order to verify the nature of the minima and to compute the total free energies of each conformer and their corresponding Boltzmann populations. All frequencies were scaled by 0.977 as recommended for that level of theory. ${ }^{66}$

QM/MMPol and QM/PCM excited state calculations, on the other hand, were performed at different QM levels of theory: semiempirical Zerner's intermediate neglect of differential overlap (ZINDO), ${ }^{67}$ configuration interaction of single excitations (CIS), ${ }^{68}$ and time-dependent density functional theory (TD-DFT) ${ }^{69}$ using the CAMB3LYP functional. ${ }^{70}$ In CIS and TD-CAMB3LYP calculations we used the 6$31 \mathrm{G}(\mathrm{d})$ basis set. All calculations were performed with a locally modified version of the Gaussian 09 code. $^{71}$ In the PCM calculations, we used the default cavity parameters in Gaussian. On the other hand, QM/MMPol calculations were performed using the Amber pol12 AL polarizable force field to describe the protein and water solvent, ${ }^{72,73}$ where water atomic charges were computed at the MP2/aug-cc-pVTZ level of theory on the TIP3P geometry using the RESP approach. Calculations for the model FBP-TrpMe dyads were performed either on the single Trp and FBP chromophoric units (the indole ring of Trp and the two phenyl rings of FBP, D-A system) or on the complete FBPTrpMe dyad including the bridge linking those units (D-B-A system). In the D-B-A models, the D/A units were separated at the bond between the $\mathrm{C}_{a}$ and the $\mathrm{N}$ atoms of TrpMe in order to compute the transition densities for each fragment. In both D-A and D-B-A model fragments, we capped the appropriate carbon atoms by introducing hydrogens at standard bond lengths. In all D-B-A and D-A monomer and coupling calculations we used the PCM cavity corresponding to the complete FBP-TrpMe molecule. 


\section{RESULTS AND DISCUSSION}

In this study, we aim at investigating the dynamic fluorescence quenching observed in FBP-TrpMe model dyads and FBP-HSA complexes by Vayá and coworkers. ${ }^{14}$ In particular, a fast stereoselective decay of the fluorescence at $310 \mathrm{~nm}$, where emission is dominated by FBP, was suggested to indicate fast energy transfer from 1FBP* to the Trp214 residue in HSA. By simulating the energy transfer dynamics in these systems, we want to validate this hypothesis and critically assess the ability of Förster theory and its underlying approximations to discriminate the FRET properties of the FBP enantiomers bound to HSA. In turn, this allows us to critically examine the theoretical binding modes simulated by MD and the potential of the MD-QM/MMPol strategy proposed to describe FRET processes in protein-ligand complexes.

\subsection{FBP-TrpMe model dyads}

The dynamic quenchings observed in (S,S)- and (R,S)-FBP-TrpMe are expected to strongly depend on the conformational preferences adopted by the dyad in acetonitrile solution. To investigate such preferences, in particular the mutual arrangement between the FBP and Trp units, we performed classical molecular dynamics (MD) simulations for each dyad. The structures sampled along a $100 \mathrm{~ns}$ MD trajectory indicated that both dyads adopt an extended conformation most of the time. In order to investigate whether or not the conformations sampled by the MD are realistic, we then determined the most stable conformations of the dyads in acetonitrile solution by performing DFT (B3LYP-D3) geometry optimizations coupled to the SMD continuum solvation model. ${ }^{65}$ In particular, we optimized the geometry of the dyads starting from a set of 100 structures extracted from the MD, optimizations which lead in many cases to equivalent conformers. Analysis of the free energies of the final unique optimized structures indicated that several stacked conformations in which the FBP and the Trp aromatic rings are in close contact mainly populate the ensemble of both dyads, whereas extended conformations are destabilized by several $\mathrm{kcal} / \mathrm{mol}$. Thus, the classical force field used in the MD was clearly misrepresenting the conformational preferences of the dyads by overstabilizing extended structures. This probably arises from an underestimation of dispersion interactions among the aromatic rings or an unbalanced description of solvation effects among extended and stacked conformations. 
We thus focused on the most stable conformations identified from DFT calculations contributing $>1 \%$ to the ensemble, which displayed a similar stacked orientation differing in i) the orientation of the Trp indole ring with respect to the FBP rings, ii) the orientation of the methyl ester group, and iii) the position of the $\mathrm{F}$ atom on one side or the other of the FBP central ring. In Fig. 1, we report these conformations, together with the corresponding Boltzmann populations, following a notation in which $\mathrm{R} / \mathrm{S}$ indicate the $(\mathrm{R}, \mathrm{S})-$ or the $(\mathrm{S}, \mathrm{S})$-enantiomer, and $\mathrm{A} / \mathrm{B}$ are used for equivalent conformations where the position of the F atom is located on opposite sides of the FBP central ring.

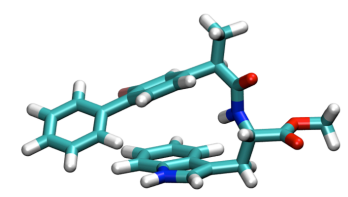

R1A $12 \%$

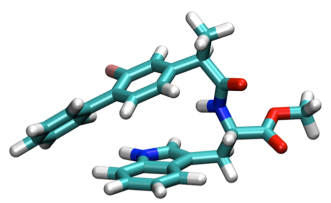

R3A $17 \%$

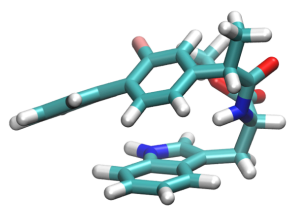

S1A $8 \%{ }^{\circ}$

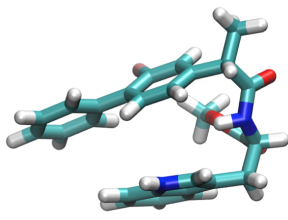

S 3 A $11 \%$

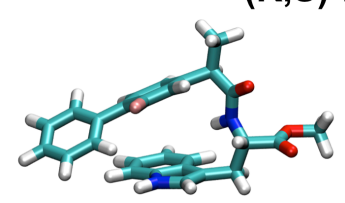

R1B 10\%

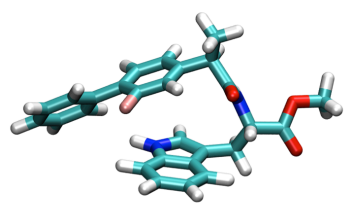

R3B 6\%

(S,S)-FBP-TrpMe

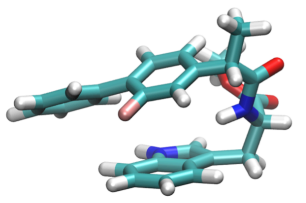

S1B $24 \%$

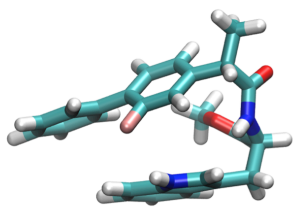

S3B $30 \%$

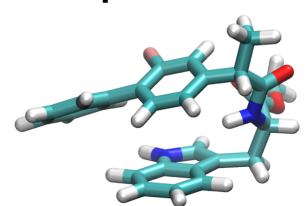

$\mathrm{S} 2 \mathrm{~A} 7 \%$

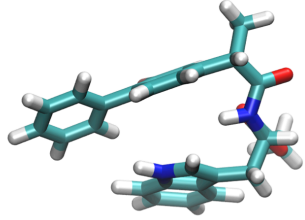

S4A $4 \%$

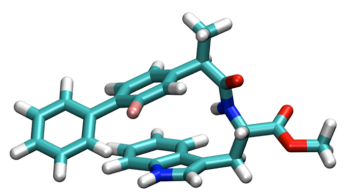

R2B 8\%

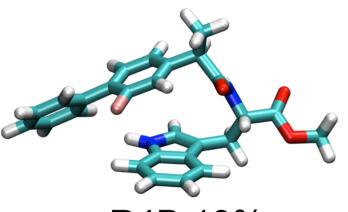

R4B 13\%

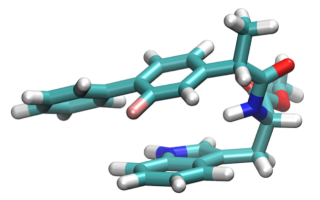

S2B $14 \%$

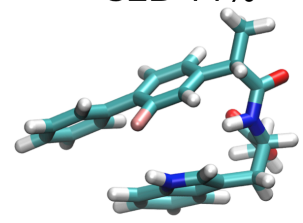

S4B 3\%

Figure 1. Main conformers for the (R,S)- and (S,S)-FBP-TrpMe dyads and their corresponding Boltzmann populations predicted by SMD-B3LYP-D3/6-31G(d) calculations in acetonitrile solution.

Accordingly, we computed the excitonic couplings for the different conformers of the dyads shown in Fig. 1 describing the surrounding acetonitrile solvent though the PCM continuum solvation model, as given by Eq. 10. In particular, we performed calculations for both D-A models, where only the aromatic rings of the chromophores are accounted for, as well as for D-B-A models, where the bridge connecting them is also included. In Table 1 we report the orientation factors and the ratios of squared 
coupling values obtained for each enantiomer, which are averaged over the Boltzmann populations of the conformers. The actual values of squared couplings, screening factors as well as the average transition energies and dipoles of FBP and Trp are reported in Tables S1-S2 of the Supporting Information, whereas in Table 2 we report the coupling data for the individual D-B-A conformers of the dyads obtained at the TD-CAMB3LYP level of theory.

In line with the absorption spectra of the dyads, ${ }^{14}$ our calculations predict almost no changes in transition energy or transition dipole strength among enantiomers, indicating that changes in energy transfer properties arise from the $V^{2}$ electronic factor in Eq. 1, instead of the spectral overlap factor $J$. Interestingly the mutual arrangement of the chromophores leads to an almost orthogonal configuration of the transition dipole moments of the $L_{a}$ state of $\operatorname{Trp}$ and the bright $\pi \rightarrow \pi^{*}$ state of FBP. If we ignore the bridge that links the Trp and the FBP chromophoric units in the calculations (D-A models), we find average squared orientation factors $\left\langle\kappa^{2}\right\rangle \sim 0.07-0.10$ and $0.13-0.18$ for the $(\mathrm{R}, \mathrm{S})$ - and $(\mathrm{S}, \mathrm{S})$-enantiomers, respectively, in contrast with the $\sim 2$ faster dynamic quenching observed for the (R,S)-diastereomer. That result is very similar regardless of whether the transition dipole moments are derived from semiempirical ZINDO, CIS or TD-DFT calculations. Because of the almost orthogonal orientation of the respective dipoles, the coupling in the complex however becomes extremely sensitive to the precise orientation of such dipoles. If we add the bridge linking the Trp and FBP units (D-B-A models), the $\operatorname{Trp} \mathrm{L}_{\mathrm{a}}$ dipole slightly reorients leading to $\mathrm{a} \sim 30 \%$ decrease in the $\left\langle\kappa^{2}\right\rangle$ value for the (S,S)-dyad, whereas it remains very similar for the (R,S) one. The resulting ratio among $\left\langle\kappa^{2}\right\rangle$ values, however, still suggest a faster energy transfer for the $(\mathrm{S}, \mathrm{S})$-enantiomer. Indeed, the ratios among squared coupling values $\left\langle V^{2}(R)\right\rangle /\left\langle V^{2}(S)\right\rangle$ shown in Table 1, obtained either using the ideal dipole approximation or from the QM/PCM model based on transition densities, are in most cases smaller than one.

As expected, our results show that Förster dipole-dipole approximation introduces significant deviations in the coupling estimates given the close separation between FBP and Trp in the dyad, especially in the D-B-A models. If we focus on the most accurate estimates based on transition densities, interestingly, only the TD-DFT data based on the complete D-B-A models is able to describe the correct enantioselectivity, whereas the simple D-A models or the CIS and ZINDO calculations predict a $\sim 2-3$ faster transfer for the $(\mathrm{S}, \mathrm{S})$-dyad, in contrast with the observed ratio 
$k_{q}(R) / k_{q}(S)=2.21$ among dynamic quenching rates experimentally observed in the dyads. ${ }^{14}$ The TD-DFT calculations, however, still predict a ratio $\left\langle V^{2}(R)\right\rangle /\left\langle V^{2}(S)\right\rangle=$ 1.12 smaller than the experimental value 2.21.

Table 1. Orientation factors and electronic couplings calculated for the (S,S)- and $(\mathrm{R}, \mathrm{S})-\mathrm{FBP}-T r p M e$ model dyads at different QM/PCM levels of theory.

$$
\left\langle\kappa^{2}\right\rangle^{\mathrm{ab}} \quad\left\langle V^{2}(R)\right\rangle /\left\langle V^{2}(S)\right\rangle^{\mathrm{a}}
$$

\section{Dip-dip $^{\mathrm{b}} \quad$ QM/PCM}

\section{ZINDO}

$\begin{array}{lllll}\text { D-A }^{c} & \text { R } & 0.10 & 0.68 & 0.25 \\ & \text { S } & 0.13 & & \\ \text { D-B-A }^{\text {d }} & \text { R } & 0.10 & 1.05 & 0.30\end{array}$

CIS

$\begin{array}{lllll}\text { D-A }^{\mathrm{c}} & \mathrm{R} & 0.07 & 0.39 & 0.36 \\ & \mathrm{~S} & 0.13 & & \\ \text { D-B-A }^{\mathrm{d}} & \mathrm{R} & 0.06 & 0.39 & 0.61\end{array}$

TD-CAMB3LYP

$\begin{array}{lllll}\text { D-A }^{\mathrm{c}} & \mathrm{R} & 0.09 & 0.44 & 0.53 \\ & \mathrm{~S} & 0.18 & & \\ \text { D-B-A }^{\mathrm{d}} & \mathrm{R} & 0.07 & 0.52 & 1.12\end{array}$

$\operatorname{Exp} k_{q}(R) / k_{q}(S)^{\mathrm{e}}$

${ }^{\mathrm{a}}$ Values averaged over the Boltzmann populations of the conformers. ${ }^{\mathrm{b}}$ Dipole-dipole couplings calculated from the transition dipoles of the corresponding QM/PCM calculations based on the center of the indole ring (Trp) and the center of the two phenyl units (FBP). ${ }^{\mathrm{c}}$ Calculations performed on the single Trp and FBP chromophoric units (aromatic rings). ${ }^{\mathrm{d}}$ Calculations performed on the complete FBP-TrpMe dyad. ${ }^{\mathrm{e}}$ Dynamic quenching rates measured from fluorescence upconversion. ${ }^{14}$ 
Here, it is worth noting that in Förster dipole-dipole picture, the ratio among enantiomers arises entirely from the changes in the orientation factors, as interchromophoric distances and transition dipole strengths are very similar, as reported in Tables S1-S2 of the Supporting Information. In the more accurate picture obtained from QM-PCM calculations, however, not only the full transition densities are considered, but also the shape of the chromophores is accounted for while evaluating screening effects. The screening factors reported in Table S2 indicate that Förster overestimates dielectric screening in the dyad, as the $s^{2}$ values range from $\sim 0.33$ to 0.53 in the D-B-A models, depending on the QM method of choice, whereas Förster approximation leads to a value $1 / n^{4}=0.31$. However, we find similar screening effects in both dyads, so solvent screening does not contribute to the observed enantioselective energy transfer.

In order to get further insights in the origin of the coupling values, in Table 2 we report the results obtained for each individual conformer of the dyads. In the case of (R,S)-FBP-TrpMe, most conformers display similar coupling values and orientation factors $\left\langle\kappa^{2}\right\rangle \sim 0.05-0.10$. In contrast, the results for the (S,S)-enantiomer clearly show two different behavious. Whereas conformers S1A/B and S2A/B are characterized by an orientation factor $\left\langle\kappa^{2}\right\rangle \sim 0.25$, the rotated orientation of the indole group of Trp in conformers $\mathrm{S} 3 \mathrm{~A} / \mathrm{B}$ and $\mathrm{S} 4 \mathrm{~A} / \mathrm{B}$ makes both transitions virtually orthogonal with orientation factors $\left\langle\kappa^{2}\right\rangle \sim 0.01$. Thus, the population of S3A/B and S4A/B conformers are the origin of the enantioselective dynamic quenching, making energy transfer in the (R,S)-enantiomer about 2 times faster. Our TD-DFT calculations, however, only predict an energy transfer $12 \%$ faster probably owing to the extreme sensitivity of our results to the relative free energies calculated for the $\mathrm{S} 3 \mathrm{~A} / \mathrm{B}$ and $\mathrm{S} 4 \mathrm{~A} / \mathrm{B}$ conformers compared to the $\mathrm{S} 1 \mathrm{~A} / \mathrm{B}$ and $\mathrm{S} 2 \mathrm{~A} / \mathrm{B}$ ones, the two groups having a population of about $50 \%$. In this case, a further stabilization of the $\mathrm{S} 3 \mathrm{~A} / \mathrm{B}$ and $\mathrm{S} 4 \mathrm{~A} / \mathrm{B}$ conformers by just $0.7 \mathrm{kcal} / \mathrm{mol}$ would increase their overall population to $75 \%$ and lead to an excellent agreement with experiments. Of course, another reason for this discrepancy could be related to the intrinsic limitations of the TD-DFT method. ${ }^{74}$ 
Table 2. Boltzmann populations, interchromophoric distances, orientation factors and electronic couplings calculated for the complete D-B-A conformers of the (S,S)- and (R,S)-FBP-TrpMe model dyads at the PCM TD-CAMB3LYP/6-31G(d) level of theory.

\begin{tabular}{|c|c|c|c|c|c|}
\hline & $\%^{\mathrm{a}}$ & $\langle R\rangle(\AA ̊)$ & $\left\langle\kappa^{2}\right\rangle$ & $\left\langle\mathrm{V}_{\text {dip-dip }}^{2}\right\rangle^{\mathrm{b}}\left(\mathrm{cm}^{-1}\right)$ & $\left\langle\mathrm{V}_{Q M / P C M}^{2}\right\rangle\left(\mathrm{cm}^{-1}\right)$ \\
\hline \multicolumn{6}{|c|}{$(\mathrm{R}, \mathrm{S})-\mathrm{FBP}-\mathrm{TrpMe}$} \\
\hline R1A & 12 & 4.09 & 0.06 & $1.50 \times 10^{4}$ & $5.04 \times 10^{4}$ \\
\hline $\mathrm{R} 1 \mathrm{~B}$ & 10 & 3.99 & 0.09 & $2.75 \times 10^{4}$ & $4.05 \times 10^{4}$ \\
\hline $\mathrm{R} 2 \mathrm{~A}$ & 19 & 4.08 & 0.05 & $1.29 \times 10^{4}$ & $4.87 \times 10^{4}$ \\
\hline $\mathrm{R} 2 \mathrm{~B}$ & 8 & 4.17 & 0.02 & $3.50 \times 10^{3}$ & $3.15 \times 10^{4}$ \\
\hline $\mathrm{R} 3 \mathrm{~A}$ & 17 & 3.97 & 0.06 & $1.92 \times 10^{4}$ & $2.42 \times 10^{4}$ \\
\hline R3B & 6 & 4.68 & 0.11 & $1.25 \times 10^{4}$ & $6.90 \times 10^{3}$ \\
\hline $\mathrm{R} 4 \mathrm{~A}$ & 15 & 3.99 & 0.05 & $1.58 \times 10^{4}$ & $2.49 \times 10^{4}$ \\
\hline $\mathrm{R} 4 \mathrm{~B}$ & 13 & 4.69 & 0.14 & $1.57 \times 10^{4}$ & $6.64 \times 10^{3}$ \\
\hline \multicolumn{6}{|c|}{$(\mathrm{S}, \mathrm{S})-\mathrm{FBP}-\mathrm{TrpMe}$} \\
\hline S1A & 8 & 3.87 & 0.25 & $7.64 \times 10^{4}$ & $4.52 \times 10^{4}$ \\
\hline S1B & 24 & 4.03 & 0.22 & $5.24 \times 10^{4}$ & $5.24 \times 10^{4}$ \\
\hline S2A & 7 & 3.94 & 0.21 & $6.05 \times 10^{4}$ & $4.29 \times 10^{4}$ \\
\hline S2B & 14 & 4.09 & 0.23 & $4.86 \times 10^{4}$ & $5.31 \times 10^{4}$ \\
\hline S3A & 11 & 4.36 & 0.02 & $3.94 \times 10^{3}$ & $4.06 \times 10^{3}$ \\
\hline S3B & 30 & 4.25 & 0.00 & $3.80 \times 10^{2}$ & $1.54 \times 10^{2}$ \\
\hline S4A & 4 & 4.91 & 0.01 & $1.38 \times 10^{3}$ & $1.37 \times 10^{4}$ \\
\hline S4B & 3 & 4.35 & 0.00 & $3.67 \times 10^{2}$ & $2.72 \times 10^{3}$ \\
\hline
\end{tabular}

${ }^{a}$ Boltzmann weights derived from free energies calculated at the SMD-B3LYP-D3/6$31 G(d)$ level of theory. ${ }^{b}$ Dipole-dipole couplings include Förster screening factor $s^{2}=1 / n^{4}=0.31\left(n^{2}=1.806\right.$ in acetonitrile $)$.

Overall, the results found for the dyad strongly support an energy transfer process from the $1 \mathrm{FBP}^{*}$ state to the $\operatorname{Trp} \mathrm{L}_{\mathrm{a}}$ state as the origin for the observed dynamic quenching. In addition, they show that capturing the proper orientation for the $\operatorname{Trp} \mathrm{L}_{\mathrm{a}}$ dipole, as well as the proper balance among $(\mathrm{S}, \mathrm{S})$-conformers, is key in order to explain 
the observed enantioselectivity. Indeed, both dipole-dipole and transition density-based estimates of the coupling values lead in most cases to a wrong answer that predict similar transfer rates for the enantiomers or even a faster transfer for the $(\mathrm{S}, \mathrm{S})$ one. Based on the spectral overlaps computed from the experimental spectra of FBP and TrpMe in acetonitrile, in Table 3 we also report a comparison between the actual values of the experimental and predicted energy transfer rates. Despite the fact that the TDDFT simulations are able to predict the enantioselectivity, although with moderate accuracy, the actual rates are too fast by approximately one order of magnitude. This discrepancy could arise from the neglect of thermal effects in the coupling values, which are very sensitive to the actual geometry of the dyad due to the almost orthogonal orientation of the transition dipoles. In addition, the significant coupling between FBP and TrpMe could lead to potential deviations from Förster ideal weak coupling regime, although in this case the fact that the absorption spectra of FBP-TrpMe is approximately the sum of the FBP and TrpMe ones supports the weak coupling assumption. ${ }^{14}$

Table 3. Electronic energy transfer times calculated at the PCM TD-CAMB3LYP/6$31 \mathrm{G}(\mathrm{d})$ level of theory for the (R,S)- and (S,S)-FBP-TrpMe dyads.

\begin{tabular}{ccccc}
\hline & & & $\tau_{F R E T}(\mathrm{ps})^{\mathrm{a}}$ & \\
& & Dip-dip $^{\mathrm{b}}$ & $\mathrm{QM}^{\mathrm{P} \mathrm{PCM}^{\mathrm{b}}}$ & Exp $^{\mathrm{c}}$ \\
\hline \multirow{2}{*}{ D-B-A } & $\mathrm{R}$ & 3.60 & 1.81 & 28 \\
& $\mathrm{~S}$ & 1.86 & 2.03 & 62 \\
\hline
\end{tabular}

${ }^{\mathrm{a}}$ Energy transfer times defined as the inverse of the rate $\tau_{F R E T}=1 / k_{F R E T}{ }^{\mathrm{b}}$ Energy transfer rates calculated using a spectral overlap $J=0.1208 \mathrm{eV}^{-1}$ obtained from the emission spectra of FBP and the absorption spectra of TrpMe in acetonitrile/air ${ }^{14}$ according to Eq. $2{ }^{\mathrm{c}}$ Dynamic quenching rates measured from fluorescence upconversion in Ref. ${ }^{14}$.

\subsection{FBP/HSA complexes}

The first question to address in order to explain the subnanosecond stereoselective dynamic quenching of FBP regards the location of the drug in the HSA complex. The experiments were performed at an FBP/HSA ratio $0.7: 1,{ }^{14}$ and at this ratio $40 \%$ and $30 \%$ of (S)- and (R)-FBP are bound to the secondary binding site of HSA, whereas the remaining drug is bound to the preferred site II. ${ }^{46}$ As discussed in the 
introduction, we argue that this secondary binding corresponds to a site of HSA at the interface between subdomains IIA and IIB. ${ }^{50}$ In contrast to the model dyads, where a $>90 \%$ quenching was observed, in the FBP/HSA complexes the quenching was around $\sim 50 \%$. We thus performed exploratory calculations of the approximate timescales of $1 F B P *$ to $\operatorname{Trp} \mathrm{L}_{\mathrm{a}}$ excitation transfer by aligning both enantiomers of FBP at the location of ibuprofen at both sites of HSA, as found in the crystal structure (PDB 2BXG) ${ }^{50}$ In this calculations, we estimated the Coulomb coupling using the full transition densities (Eq. 8) obtained at the ZINDO level and accounted for dielectric screening through a simple Förster screening factor $1 / n^{2}$ (Eq. 4), where $n^{2}$ was taken to be 2 for a protein environment. Then, we computed a spectral overlap factor $J=0.1321 \mathrm{eV}^{-1}$ from the emission spectra of FBP and the absorption spectra of $\mathrm{HSA}^{14}$ according to Eq. 2. The estimated energy transfer rates for (R)- and (S)-FBP to Trp214 energy transfer were found to be $\sim 500-800$ ps for the enantiomers located at the secondary site, and much slower, $\sim 30-150 \mathrm{~ns}$ when located at site II, in line with the much shorter distance $\sim 10 \AA$ between FBP and Trp214 in the secondary site compared to $\sim 20 \AA$ in site II. The timescales predicted from the secondary site are in excellent agreement with the subnanosecond quenching times of 180 and 250 ps measured for (S)-FBP and (R)-FBP, whereas the rates from site II are clearly too slow. This explains why only a $\sim 50 \%$ quenching of FBP fluorescence is observed, as only the fraction of FBPs located in the secondary site contribute to the subnanosecond dynamic quenching through energy transfer to Trp214. We have thus focused only on the binding of FBP at the secondary site of HSA, which is postulated as the origin of the dynamic quenching.

In the FBP/HSA complexes, as in the model dyads discussed above, the energy migration properties between the FBP enantiomers and the Trp214 exquisitely depend on the separation and mutual orientation between them. We have thus run MD simulations for a total time of $0.5 \mu \mathrm{s}$, divided in 5 replicas, for each enantiomer of FBP. In Fig. S1 of the Supporting information we show the root-mean-squared deviations (RMSD) of atomic positions for the complex and for the ligand along each MD replica. As can be observed from the RMSD plots, the ligand is rather flexible in the binding pocket. In particular the (S)-FBP enantiomer often changes its configuration in the binding site, enlarging the RMSD values to $\sim 4-5 \AA$, although rapidly it returns to the stable binding mode with an RMSD of $\sim 2-3 \AA$. Such transitions are also observed for the $(\mathrm{R})$-enantiomer, although to a smaller extent. Overall, the ligand and the protein RMSD values indicate that the trajectories are stable. 


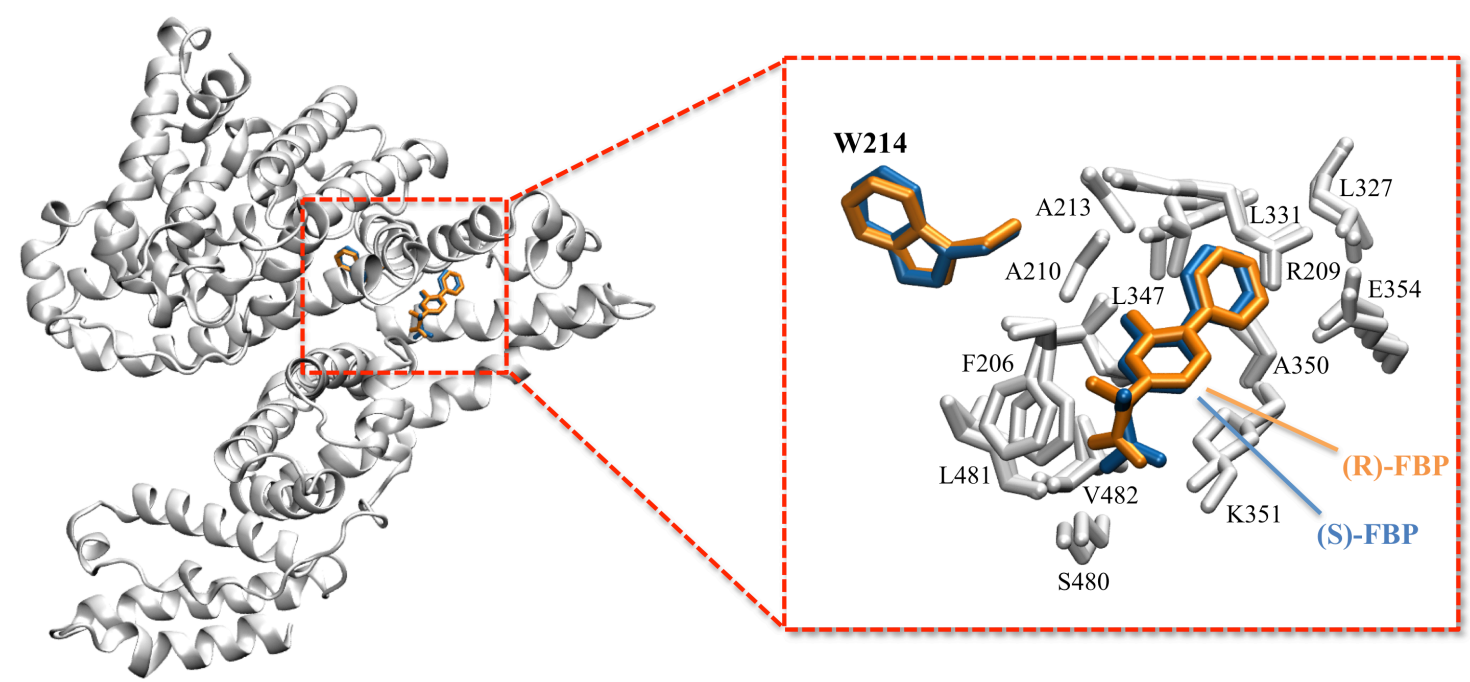

Figure 2. Illustration of the binding modes predicted from MD simulations for the (R)and (S)-enantiomers of FBP at the secondary site of HSA located at the interface between subdomains IIA and IIB. The inset shows the FBP ligand, the Trp214 residue participating in the energy transfer process, and the amino acids surrounding the ligand in the binding pocket.

In Fig. 2, we show the arrangement of (R) and (S)-FBP in the secondary binding site of HSA at the end of the trajectories. As can be observed, the binding mode predicted for both enantiomers is very similar. Moreover, the mutual orientation between FBP and Trp214 is also comparable, thus at first sight unable to explain the $30 \%$ faster quenching observed in the fluorescence of (S)-FBP compared to the other enantiomer. However, as indicated by the RMSD plots, the ligands are rather flexible, and such flexibility could have a strong impact on the actual electronic coupling between Trp214 and FBP. In Fig. 3 we show the fluctuations of the electronic couplings, calculated at the QM/MMPol TD-CAMB3LYP/6-31G(d) level of theory, for each enantiomer and MD replica. As can be observed, there are large fluctuations around the average coupling values. Indeed, the standard deviations found for the coupling fluctuations, $\sigma \sim 5 \mathrm{~cm}^{-1}$, are comparable to the mean values $\langle V\rangle \sim 7 \mathrm{~cm}^{-1}$ for both enantiomers. Such dramatic oscillations, for example, are in sharp contrast to results found for photosynthetic pigment-protein complexes, where the pigments are tightly held to the proteins scaffold and standard deviations typically amount to only $\sim 20 \%$ of the mean coupling values. ${ }^{18}$ Thus, whereas in photosynthetic complexes it is 
common to ignore coupling fluctuations and evaluate the pigment-pigment interactions based on the crystal structure, ${ }^{42}$ such strategy is clearly not justified in the case of a drug-protein complex, where the bound ligand has more flexibility, even if that flexibility depends on each particular ligand and binding site. We thus average the coupling values over the MD trajectories. Note, however, that we do not explicitly account for the coupling fluctuations in the estimate of FRET rates. Explicit account of such fluctuations has been recently shown to introduce negligible changes in the exciton dynamics of a photosynthetic complex. ${ }^{75}$ However, when the chromophores involved in the transfer display a larger degree of structural dynamics, as happens in common FRET experiments, it can be important to explicitly account for such coupling fluctuations, for example, by processing the coupling trajectory through Markov chain approaches. ${ }^{29,34}$ Here, the adoption of an average coupling value is deemed a reasonable approximation, given that the FBP ligand stays most of the time in a stable binding mode.

a)

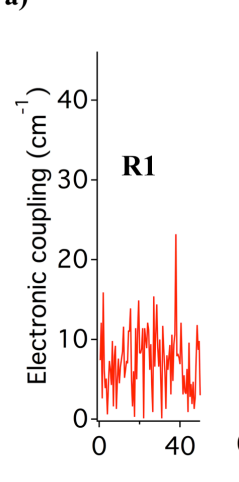

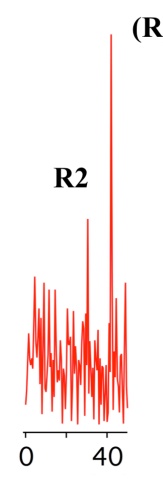

(R)-FBP/HSA

R3 R4 R5
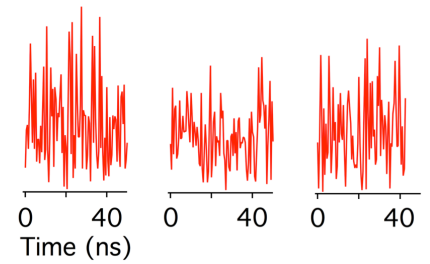

b)

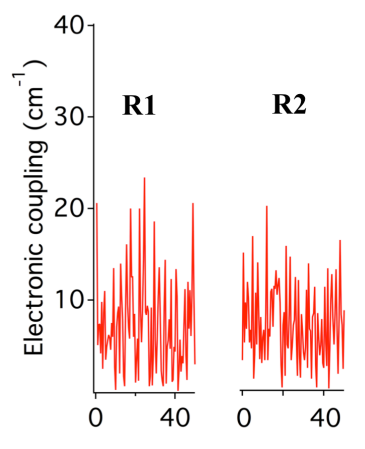

(S)-FBP/HSA

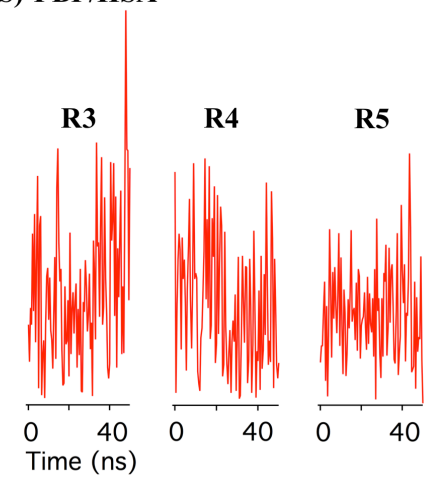

Figure 3: Fluctuations of electronic couplings along the $5 \mathrm{MD}$ replicas calculated at the QM/MMPol TD-CAMB3LYP/6-31G(d) level of theory between Trp214 and the (R)and (S)-enantiomers of FBP in the HSA complex. 


\section{(R)-FBP/HSA}
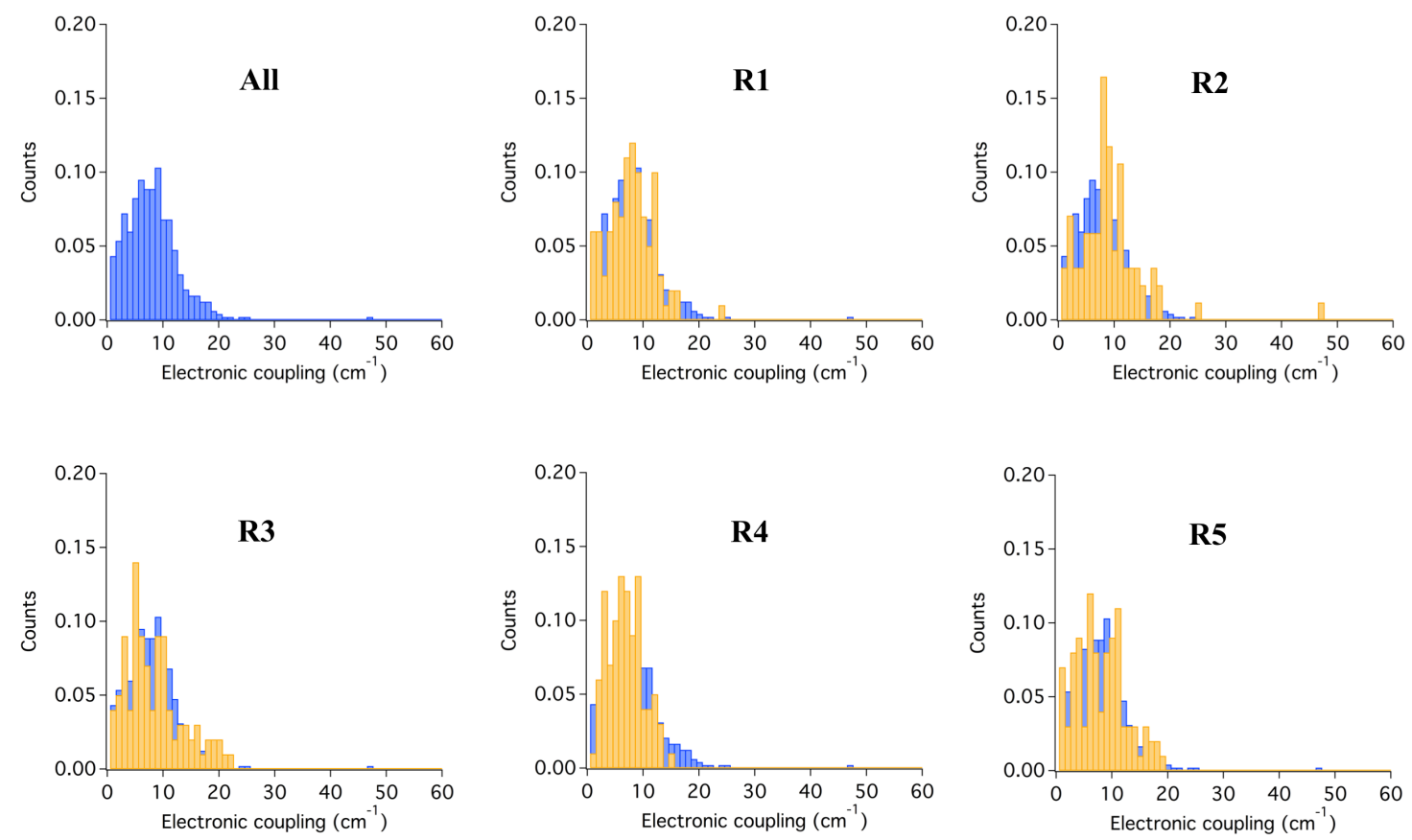

Figure 4. Distribution of electronic couplings calculated at the MD-QM/MMPol TDCAMB3LYP/6-31G(d) level of theory for the (R)-FBP/HSA complex: total distribution (blue) and distributions computed for each MD replica (yellow).

(S)-FBP/HSA
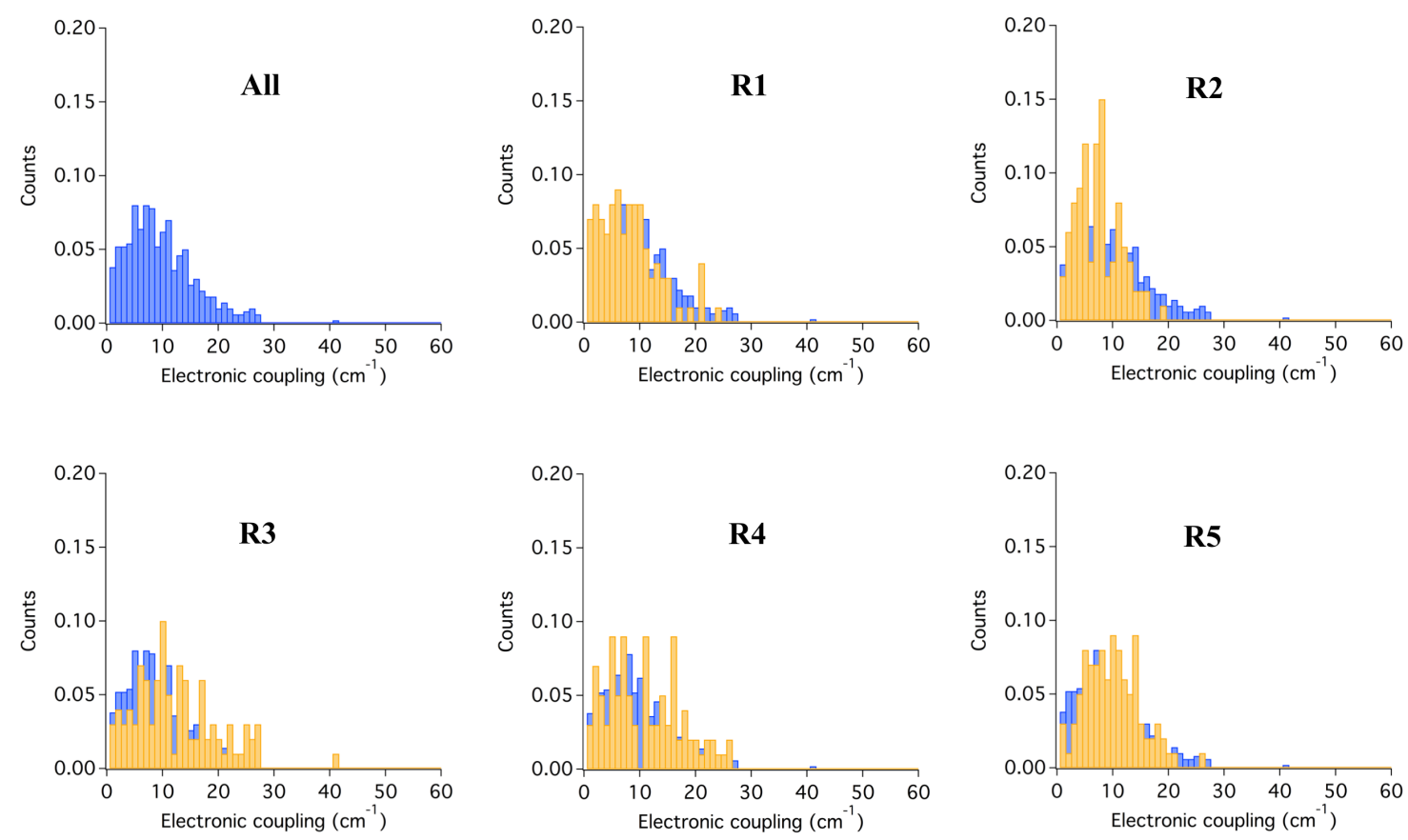

Figure 5. Distribution of electronic couplings calculated at the MD-QM/MMPol TDCAMB3LYP/6-31G(d) level of theory for the (S)-FBP/HSA complex: total distribution (blue) and distributions computed for each MD replica (yellow). 
Thus, the large degree of coupling fluctuations makes it necessary to average the energy transfer properties over a large number of structures. In Fig. 4 and 5 we show the total distributions of coupling values, as well as those derived from each MD replica. As can be observed, the total distributions, which include a total of 500 structures extracted along the $0.5 \mu \mathrm{s}$ of $\mathrm{MD}$, are reasonably well converged, whereas the individual distributions derived for the individual replicas are clearly not representative of the ensemble. Interestingly, the distribution of coupling values has a similar maximum around $\sim 7-8 \mathrm{~cm}^{-1}$ for both enantiomers, but the (S)- one displays a larger tail in the distribution toward larger values, thus leading to a mean coupling $\langle V\rangle=9.2 \mathrm{~cm}^{-1}$ compared to the smaller value $7.4 \mathrm{~cm}^{-1}$ found for (R)-FBP. The tail toward large coupling values indicates a the larger degree of flexibility of the (S)-FBP diastereomer compared to the (R)- one, in line with the larger RMSD fluctuations from our simulations discussed previously and the faster reorientational time derived from fluorescence anisotropy. ${ }^{14}$ We have analyzed the RMSD values of the structures that give rise to the tail toward high-coupling values, that is, those leading to coupling $>22$ $\mathrm{cm}^{-1}$, and found no particular increase in the RMSD values for the high-coupling structures. Thus, the temporary changes in binding mode, illustrated by $\sim 4-5 \AA$ RMSD values, are not the reason for the increased coupling strength. Rather, it is the dynamic characteristics of the stable binding mode characterized by RMSD of $\sim 2-3 \AA$ that lead to the wider coupling distribution for the (S)-enantiomer.

In order to get further insights in the origin of the larger coupling distribution obtained for (S)-FBP, in Table 4 we provide the corresponding FBP-Trp214 distances and orientation factors, as well as the ratio $\left\langle V^{2}(S)\right\rangle /\left\langle V^{2}(R)\right\rangle$, calculated at the ZINDO, CIS and TD-CAMB3LYP levels of theory. The corresponding values of transition energies and dipoles, as well as the averaged values of squared electronic couplings and screening factors are provided in Tables S3-S4 of the Supporting information. As in the model dyads discussed in the previous section, we find no significant differences in transition energies or dipole strengths fort the states of FBP and Trp in the (R)- and (S)FBP/HSA complexes, in agreement with the similar absorption spectra measured for both complexes. ${ }^{14}$ Thus, variations in the $1 \mathrm{FBP}^{*}$ to Trp energy transfer arise from the $V^{2}$ electronic factor in Eq. 1, as the spectral overlap factor $J$ does not change. The $\left\langle V^{2}(S)\right\rangle /\left\langle V^{2}(R)\right\rangle$ ratios predicted from our QM/MMPol simulations indeed nicely explain the ratio of quenching rates $k_{q}(S) / k_{q}(R)$ measured from Fluorescence 
Upconversion, indicating a $89 \%, 77 \%$ and $54 \%$ faster energy transfer for the (S)enantiomer at the ZINDO, CIS and TD-DFT levels of theory. Thus, as expected, and in line with the findings discussed in the previous section for the model FBP-TrpMe dyads, again TD-DFT performs slightly better than ZINDO or CIS by predicting a $54 \%$ faster quenching for (S)-FBP compared to the (R) form, very close to the $30 \%$ experimental value. The better performance of TD-DFT is even amplified when the couplings are derived based on the ideal dipole approximation, as in this case only the dipole data derived from TD-DFT is able to explain the experimental ratio giving a rather accurate estimate of $38 \%$ increase, whereas the adoption of transition dipoles derived from CIS and ZINDO calculations predict faster rates in the order of $214 \%$ and $489 \%$. The important conclusion from this data is that the dipole-dipole couplings assumed in Förster theory are too sensitive to small changes in the actual orientation of the transition dipoles, whereas the estimates based on full 3D transition densities are remarkably more robust to such changes and make the choice of QM method less critical.

If we turn again to the origin of the larger coupling distribution obtained for the (S)-enantiomer of FBP, the data in Table 4 allows us to dissect the variation of the coupling values in terms of distances and orientation factors. In addition, in Fig. 6 we report the distribution of screening factors derived from MD-QM/MMPol calculations compared to the $s=1 / n^{2}$ Förster approximation computed assuming a value $n^{2}=2$ for the protein environment. ${ }^{18}$ Regarding the Trp214 to FBP distances, we find a slightly shorter average separation $10.5 \AA$ for (S)-FBP compared to the value 11.0 $\AA$ found for (R)-FBP. Such change suggests a larger FRET rate for the (S)-enantiomer by $31 \%$ based on Förster $\mathrm{R}^{-6}$ distance-dependent rate expression, very close to the $30 \%$ increase observed. Other factors beyond chromophore separation however come into play, namely the orientation between chromophores and changes in dielectric screening effects. Based on the most accurate TD-DFT data, these two effects, in contrast to the FBP-Trp distance, tend to increase the FRET rate of the (R)-enantiomer by $8 \%$ each, as $\left\langle\kappa^{2}\right\rangle$ passes from 0.26 to 0.28 and $\left\langle s^{2}\right\rangle$ from 0.35 to 0.38 (see Table S4 in the Supporting Information). Thus, the average conformation of the (S)-enantiomer in the binding pocket explains only about $15 \%$ of the total $54 \%$ increase in transfer rate compared to the (R)-enantiomer as predicted by the TD-DFT data based on transition densities, an increase caused by a shorter interchromophoric distance, but counteracted by a less favorable orientation and modulation of the coupling by dielectric screening 
effects. The remaining increase, thus, can be associated to the dynamic characteristics of the stable binding mode adopted by the (S)-FBP, which seems to be more flexible, thus leading to a wider distribution of coupling values as shown in Fig. 5 and discussed before. Indeed, the distance fluctuations for the (S)-enantiomer, with a $\sigma=0.88 \AA$, are significantly larger than the value $0.56 \AA$ found for the (R) form. It is also interesting to note the large distribution of screening factors obtained along the MD simulations shown in Fig. 6, which underscore the strong variation that can arise in transfer rates due to differences in local environment and pigment orientation, an effect completely ignored when the Förster $1 / n^{2}$ screening factor is assumed.

Table 4. Interchromophoric distances, orientation factors and electronic couplings calculated for the (S)- and (R)-FBP/HSA complexes at different QM/MMPol levels of theory.

\begin{tabular}{|c|c|c|c|c|}
\hline & \multirow[t]{2}{*}{$\langle R\rangle(\AA ̊)$} & \multirow[t]{2}{*}{$\left\langle\kappa^{2}\right\rangle^{\mathrm{ab}}$} & \multicolumn{2}{|c|}{$\left\langle V^{2}(S)\right\rangle /\left\langle V^{2}(R)\right\rangle^{\mathrm{a}}$} \\
\hline & & & Dip-dip ${ }^{b}$ & QM/MMPol \\
\hline \multicolumn{5}{|l|}{ ZINDO } \\
\hline $\mathrm{R}$ & $11.01 \pm 0.56$ & $0.12 \pm 0.14$ & \multirow{2}{*}{4.89} & \multirow{2}{*}{1.89} \\
\hline $\mathrm{S}$ & $10.52 \pm 0.88$ & $0.27 \pm 0.31$ & & \\
\hline \multicolumn{5}{|l|}{ CIS } \\
\hline $\mathrm{R}$ & $11.01 \pm 0.56$ & $0.23 \pm 0.20$ & \multirow{2}{*}{2.14} & \multirow{2}{*}{1.77} \\
\hline $\mathrm{S}$ & $10.52 \pm 0.88$ & $0.26 \pm 0.28$ & & \\
\hline \multicolumn{5}{|c|}{ TD-CAMB3LYP } \\
\hline $\mathrm{R}$ & $11.01 \pm 0.56$ & $0.28 \pm 0.24$ & \multirow{2}{*}{1.38} & \multirow{2}{*}{1.54} \\
\hline $\mathrm{S}$ & $10.52 \pm 0.88$ & $0.26 \pm 0.27$ & & \\
\hline \multicolumn{3}{|c|}{$\operatorname{Exp} k_{q}(S) / k_{q}(R)^{\mathrm{c}}$} & \multicolumn{2}{|c|}{1.39} \\
\hline
\end{tabular}

${ }^{a}$ Values averaged over the MD trajectories. For distances and orientation factors, standard deviations are also reported. ${ }^{b}$ Dipole-dipole couplings calculated from the transition dipoles of the corresponding QM/MMPol calculations based on the center of the indole ring (Trp) and the center of the two phenyl units (FBP). ${ }^{c}$ Dynamic quenching rates measured from fluorescence upconversion. ${ }^{14}$ 

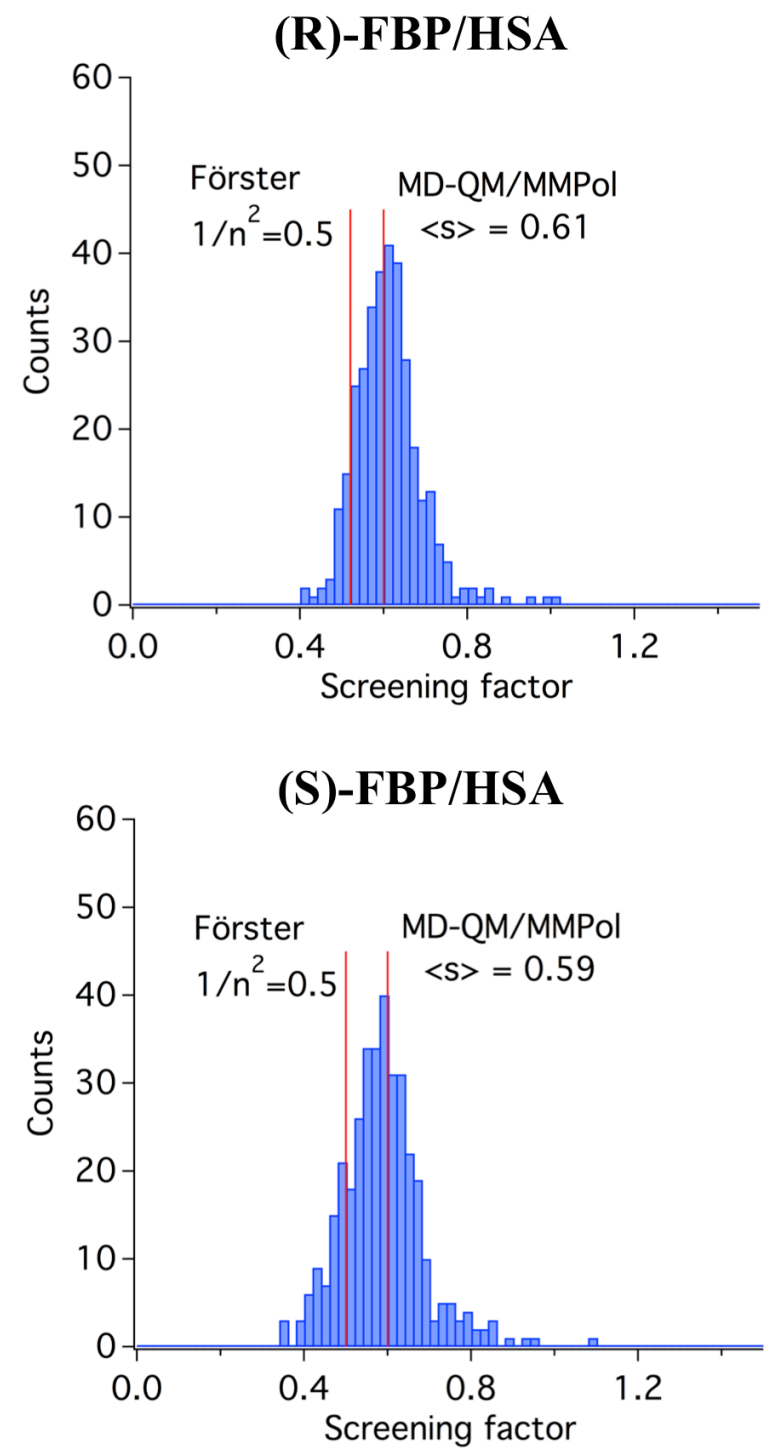

Figure 6. Distribution of dielectric screening factors calculated at the MD-QM/MMPol TD-CAMB3LYP/6-31G(d) level of theory for the (R)- and (S)-FBP/HSA complexes.

Finally, in Table 5 we provide the predicted energy migration rates for the (R)and (S)-FBP enantiomers, estimated using the MD-QM/MMPol couplings at the ZINDO, CIS and TD-CAMB3LYP levels of theory, together with the experimental spectral overlap factor. As can be seen, although TD-DFT provides the best enantioselective quenching ratios shown in Table 4, CIS and ZINDO methods in this case provide a slightly better estimate of the energy transfer times. In particular, TDDFT predicts the energy transfer to occur in 666 and 434 ps for (R)- and (S)-FBP, respectively, compared to measured quenching times of 250 and $180 \mathrm{ps},{ }^{14}$ whereas ZINDO and CIS predict values of $\sim 300-400$ and $\sim 150-200$ ps. Here, we remark that the comparison of predicted absolute FRET times with measured quenching times is 
considerably more delicate than the comparison of the relative rate among enantiomers. First, deviations in the transfer rates are expected to arise from inaccuracies in the predicted transition dipole strengths of FBP and Trp that impact the actual coupling values. Indeed, whereas TD-DFT, and in particular the CAM-B3LYP functional, is expected to provide reasonable estimates of oscillator strenghts, ${ }^{76}$ CIS has the wellknown trend to overestimate transition dipoles - and thus the electronic couplings and transfer rates, ${ }^{77}$ in line with the observed faster rates obtained with CIS compared to TD-DFT for the FBP/HSA complexes. Here we do not attempt to compare to experiments our predicted transition dipole strengths of FBP and Trp, reported in the Supporting Information, by two reasons. The main one is that the $\operatorname{Trp} \mathrm{L}_{\mathrm{a}}$ band overlaps with the weaker $\mathrm{L}_{b}$ absorption band, making experimental derivation of the $\mathrm{L}_{\mathrm{a}}$ transition dipole strength from the intensity of the absorption band not direct. In addition, the comparison between theory and experiment is not straightforward even if the band is well characterized, as local field effects need to be accounted for and different models lead to significant differences. ${ }^{78,79}$

Another reason that could explain the somewhat slow transfer times predicted by TD-DFT, which otherwise predicts the most accurate enantioselective ratio, is the potential participation of the $\operatorname{Trp} \mathrm{L}_{b}$ state in the transfer, which could slightly increase the FRET rates. In this study, we also computed the couplings of the FBP $\pi \rightarrow \pi^{*}$ state with the $\mathrm{L}_{\mathrm{b}}$ state of Trp (data not shown), which indicated that such transfer should occur 2-6 times slower that transfer to the $\operatorname{Trp} L_{a}$ state based on the coupling values. However, derivation of the spectral overlap for this weak band, which overlaps with the brighter $\mathrm{L}_{\mathrm{a}}$ band, is not clear. In addition, the experimental quenching times 250/180 ps correspond to the fastest contribution of the multiexponential fluorescence decay of the $\mathrm{FBP} / \mathrm{HSA}$ complexes at $310 \mathrm{~nm}$, where the fluorescence mainly originates from FBP, with additional slower components at $\sim 0.7$ and $\sim 3 \mathrm{~ns}$, so transfer to the $\mathrm{L}^{\mathrm{b}}$ state most probably affects such slower components. Interestingly, the average lifetimes accounting for all components in the multiexponential decay, 700 and 540 ps, are in excellent agreement with the TD-DFT predicted rates, and still describe a quenching $30 \%$ faster for the (S)-enantiomer, very similar to the ratio $38 \%$ derived only from the fastest components.

Overall, this analysis illustrates the fact that it is rather difficult to predict absolute accurate transfer times for a ligand-protein complex. The prediction of relative transfer times among the different enantiomers in a binding site, or for a given ligand in 
different binding sites, appear however to be much more robust, as the uncertainties related to the prediction of transition dipole moments - and thus in the coupling estimates - are expected to cancel in that case.

Nevertheless, our simulations strongly support a picture where energy transfer from FBP bound at the secondary site of HSA originates the subnanosecond enantioselective dynamic quenching observed. We find that the binding modes for both enantiomers at this site of HSA are fairly similar, the most apparent change in the binding pocket being a displacement of Phe206 due to the different orientation of the carboxylic group of FBP. Such similar binding modes, however, translate into a significantly larger flexibility of the (S)-enantiomer of FBP, and this appears to be the main reason for the enantioselective dynamic quenching observed..$^{14}$

Table 5. Electronic energy transfer times calculated for the (R)- and (S)-FBP/HSA complexes at different QM/MMPol levels of theory.

\begin{tabular}{|c|c|c|c|c|c|c|}
\hline & \multicolumn{6}{|c|}{$\tau_{F R E T}(\mathrm{ps})^{\mathrm{a}}$} \\
\hline & \multicolumn{3}{|c|}{ (R)-FBP/HSA } & \multicolumn{3}{|c|}{ (S)-FBP/HSA } \\
\hline & Dip-dip ${ }^{b}$ & $\mathrm{QM} / \mathrm{MMPol}^{\mathrm{b}}$ & $\operatorname{Exp}^{c}$ & Dip-dip ${ }^{b}$ & $\mathrm{QM} / \mathrm{MMPol}^{\mathrm{b}}$ & $\operatorname{Exp}^{c}$ \\
\hline ZINDO & 316 & 301 & & 64 & 159 & \\
\hline CIS & 354 & 398 & 250 & 166 & 225 & 180 \\
\hline TD-CAMB3LYP & 403 & 666 & & 293 & 434 & \\
\hline \multicolumn{7}{|c|}{$\begin{array}{l}\text { Energy transfer times defined as the inverse of the rate } \tau_{F R E T}=1 / k_{F R E T}{ }^{\mathrm{b}} \text { Energy } \\
\text { transfer rates calculated using a spectral overlap } J=0.1321 \mathrm{eV}^{-1} \text { obtained from the } \\
\text { emission spectra of FBP and the absorption spectra of } \mathrm{HSA}^{14} \text { according to Eq. } 2\end{array}$} \\
\hline
\end{tabular}

\section{CONCLUSION}

In this contribution we have explored the reliability of Förster theory in order to describe electronic energy transfer in ligand-protein complexes. We investigated the enantioselective dynamic quenching observed for the (R)- and (S)-enantiomers of FBP complexes with HSA, as well as parallel observations in a solvated model FBP-TrpMe dyad, by combining MD simulations, and QM/PCM and QM/MMPol multiscale calculations of the energy transfer properties of the system. Our results strongly support the hypothesis that the subnanosecond dynamic quenching in this systems originates 
from energy transfer from the $1 \mathrm{FBP}^{*}$ state to the $\mathrm{L}_{\mathrm{a}}$ state of Trp. In the model dyads, we show that the enantioselectivity arises from a virtually orthogonal arrangement of the transitions of FBP and Trp in several conformers of (S)-FBP-TrpMe in acetonitrile solution. In FBP/HSA complexes, our simulations show that such transfer occurs from the fraction of FBP molecules bound to the secondary site of HSA located at the interface between subdomains IIA and IIB, and that fairly similar binding modes for both enantiomers are found, such enantioselective energy transfer mostly arising from the increased flexibility of (S)-FBP in the binding pocket compared to (R)-FBP.

We show that the MD-QM/MMPol strategy is able to describe with almost quantitative accuracy the enantioselectivity in the FBP/HSA complexes and the model dyads, when a TD-DFT description is used to model the FBP and Trp excitations. This seems to be particularly important in order to describe the proper orientation of the $\mathrm{L}_{\mathrm{a}}$ transition dipole of Trp. In turn, they show that accounting for the shape of the interacting chromophores and the local environment in the excitonic couplings through the QM/MMPol model based on transition densities provides significantly more robust estimates of the transfer rates than the Förster ideal dipole approximation, where the molecular shape of the chromophores is ignored both in the calculation of the Coulomb term of the coupling as well as in the estimation of screening effects. For example, by using transition densities we find a relatively good enantioselective quenching ratio among enantiomers at ZINDO, CIS and TD-DFT levels of theory, whereas only dipoledipole estimates based on TD-DFT calculations provide results of similar quality.

Compared to photosynthetic pigment-protein complexes, where rather detailed theoretical studies on energy transfer have been presented in the last decade, we show that fluctuations in electronic couplings are much larger in a ligand-protein complex due to the considerable flexibility of the ligand, at least in the FBP/HSA complex. This finding makes it important to average coupling predictions over the thermal motions of the protein-ligand complex, whereas calculations based on a static structure, for instance a docking pose predicted for a ligand, are expected to introduce too much noise in the predictions. Regarding dielectric screening effects, we find large thermal fluctuations that translate into strong changes in the corresponding couplings. Although the average values found are quite consistent with Förster simple $1 / n^{2}$ factor, such fluctuations illustrate the fact that screening can vary significantly depending on the local environment and the donor-acceptor orientation, a factor neglected in Förster theory that 
can translate into wrong structural interpretations of FRET data that arise from changes in dielectric screening.

Overall, this study supports the potential of theoretical simulations in order to link variations in the fluorescence of ligand-protein complexes to subtle changes in the underlying structure originated by energy transfer, simulations that can be used to characterize ligand binding events both from a static and dynamic perspective at the molecular level.

\section{SUPPORTING INFORMATION}

Root-mean-squared deviation of atomic positions along the MD simulations of FBP/HSA complexes. Tables of transition energies, transition dipoles, interchromophoric distances, orientation factors, screening factors and electronic couplings for the model dyads and FBP/HSA complexes. Force field parameters derived for (R)- and (S)-FBP and for (R,S)- and (S-S)-FBP-TrpMe.

\section{ACKNOWLEDGEMENTS}

We are grateful to the Consorci de Serveis Universitaris de Catalunya for providing access to computational resources. Financial support from the Agència de Gestió d'Ajuts Universitaris i de Recerca from Generalitat de Catalunya (GENCAT; SGR2014-1189) and from the Spanish Ministerio de Economía y Competitividad (MINECO; grants RYC2011-08918 and CTQ2012-36195) are acknowledged. C. C. is a Serra Húnter Fellow. S. P. is a fellow of the Ciências Sem Fronteiras program of the Conselho Nacional de Desenvolvimento Ciêntifico e Tecnológico (CNPq) de Brasil (246791/2012-8).

\section{References}

(1) Protein Fluorescence; Lakowicz, J. R., Ed.; Topics in Fluorescence Spectroscopy; Kluwer Academic Publishers: New York, 2002; Vol. 6.

(2) Royer, C. A. Probing Protein Folding and Conformational Transitions with Fluorescence. Chem. Rev. 2006, 106, 17691784.

(3) Michalet, X.; Weiss, S.; Jäger, M. Single-Molecule Fluorescence Studies of Protein Folding and Conformational Dynamics. Chem. Rev. 2006, 106, 1785-1813.

(4) Schuler, B.; Eaton, W. A. Protein Folding Studied by Single-Molecule FRET. Curr. Opin. Struct. Biol. 2008, 18, 16-26.

(5) Ciruela, F. Fluorescence-Based Methods in the Study of Protein-Protein Interactions in Living Cells. Curr. Opin. Biotechnol. 2008, 19, 338-343.

(6) Yengo, C. M.; Berger, C. L. Fluorescence Anisotropy and Resonance Energy Transfer: Powerful Tools for Measuring Real Time Protein Dynamics in a Physiological Environment. Curr. Opin. Pharmacol. 2010, 10, 731-737. 

Spectroscopy. Phys. Chem. Chem. Phys. 2014, 16, 11139.

(8) Ghisaidoobe, A.; Chung, S. Intrinsic Tryptophan Fluorescence in the Detection and Analysis of Proteins: A Focus on Förster Resonance Energy Transfer Techniques. Int. J. Mol. Sci. 2014, 15, 22518-22538.

(9) Banerjee, P. R.; Deniz, A. A. Shedding Light on Protein Folding Landscapes by Single-Molecule Fluorescence. Chem. Soc. Rev. 2014, 43, 1172-1188.

(10) Czar, M. F.; Jockusch, R. A. Sensitive Probes of Protein Structure and Dynamics in Well-Controlled Environments: Combining Mass Spectrometry with Fluorescence Spectroscopy. Curr. Opin. Struct. Biol. 2015, 34, 123-134.

(11) Callis, P. R. Binding Phenomena and Fluorescence Quenching. I: Descriptive Quantum Principles of Fluorescence Quenching Using a Supermolecular Approach. J. Mol. Struct. 2014, 1077, 14-21.

(12) Callis, P. R. Binding Phenomena and Fluorescence Quenching. II: Photophysics of Aromatic Residues and Dependence of Fluorescence Spectra on Protein Conformation. J. Mol. Struct. 2014, 1077, $22-29$.

(13) Callis, P. R. Simulating Electrostatic Effects on Electronic Transitions in Proteins. Mol. Simul. 2015, 41, 190-204.

(14) Vayá, I.; Bonancía, P.; Jiménez, M. C.; Markovitsi, D.; Gustavsson, T.; Miranda, M. a. Excited State Interactions between Flurbiprofen and Tryptophan in Drug-protein Complexes and in Model Dyads. Fluorescence Studies from the Femtosecond to the Nanosecond Time Domains. Phys. Chem. Chem. Phys. 2013, 15, 4727.

(15) Beljonne, D.; Curutchet, C.; Scholes, G. D.; Silbey, R. J. Beyond Förster Resonance Energy Transfer in Biological and Nanoscale Systems. J. Phys. Chem. B 2009, 113, 6583-6599.

(16) VanBeek, D. B.; Zwier, M. C.; Shorb, J. M.; Krueger, B. P. Fretting about FRET: Correlation between Kappa and R. Biophys. J. 2007, 92, 4168-4178.

(17) Munoz-Losa, A.; Curutchet, C.; Hartsell, L. R.; Krueger, B. P.; Mennucci, B. Fretting About FRET: Failure of the Ideal Dipole Approximation. Biophys. J. 2009, 96, 4779-4788.

(18) Curutchet, C.; Kongsted, J.; Muñoz-Losa, A.; Hossein-Nejad, H.; Scholes, G. D.; Mennucci, B. Photosynthetic LightHarvesting Is Tuned by the Heterogeneous Polarizable Environment of the Protein. J. Am. Chem. Soc. 2011, 133, 3078 3084.

(19) Schuler, B.; Lipman, E. A.; Steinbach, P. J.; Kumke, M.; Eaton, W. A. Polyproline and the "Spectroscopic Ruler" Revisited with Single-Molecule Fluorescence. Proc. Natl. Acad. Sci. 2005, 102, 2754-2759.

(20) Jean, J. M.; Krueger, B. P. Structural Fluctuations and Excitation Transfer between Adenine and 2-Aminopurine in Single-Stranded Deoxytrinucleotides. J. Phys. Chem. B 2006, 110, 2899-2909.

(21) Beierlein, F. R.; Othersen, O. G.; Lanig, H.; Schneider, S.; Clark, T. Simulating FRET from Tryptophan: Is the Rotamer Model Correct? J. Am. Chem. Soc. 2006, 128, 5142-5152.

(22) Merchant, K. A.; Best, R. B.; Louis, J. M.; Gopich, I. V; Eaton, W. A. Characterizing the Unfolded States of Proteins Using Single-Molecule FRET Spectroscopy and Molecular Simulations. Proc. Natl. Acad. Sci. U. S. A. 2007, 104, 15281533.

(23) Dolghih, E.; Roitberg, A. E.; Krause, J. L. Fluorescence Resonance Energy Transfer in Dye-Labeled DNA. J. Photochem. Photobiol. A Chem. 2007, 190, 321-327.

(24) Wozniak, A. K.; Schroeder, G. F.; Grubmueller, H.; Seidel, C. A. M.; Oesterhelt, F. Single-Molecule FRET Measures Bends and Kinks in DNA. Proc. Natl. Acad. Sci. U. S. A. 2008, 105, 18337-18342.

(25) Allen, L. R.; Paci, E. Orientational Averaging of Dye Molecules Attached to Proteins in Förster Resonance Energy Transfer Measurements: Insights from a Simulation Study. J. Chem. Phys. 2009, 131, 1-6.

(26) Dolghih, E.; Ortiz, W.; Kim, S.; Krueger, B. P.; Krause, J. L.; Roitberg, A. E. Theoretical Studies of Short Polyproline Systems: Recalibration of a Molecular Ruler. J. Phys. Chem. A 2009, 113, 4639-4646.

(27) Stevens, J. a.; Link, J. J.; Kao, Y. T.; Zang, C.; Wang, L.; Zhong, D. Ultrafast Dynamics of Resonance Energy Transfer in Myoglobin: Probing Local Conformation Fluctuations. J. Phys. Chem. B 2010, 114, 1498-1505.

(28) Callis, P. R. Predicting Fluorescence Lifetimes and Spectra of Biopolymers, 1st ed.; Elsevier Inc., 2011 ; Vol. 487.

(29) Speelman, A. L.; Muñoz-Losa, A.; Hinkle, K. L.; VanBeek, D. B.; Mennucci, B.; Krueger, B. P. Using Molecular Dynamics and Quantum Mechanics Calculations to Model Fluorescence Observables. J. Phys. Chem. A 2011, 115, 39974008.

(30) Beauchamp, K. A.; Ensign, D. L.; Das, R.; Pande, V. S. Quantitative Comparison of Villin Headpiece Subdomain Simulations and Triplet - Triplet Energy Transfer Experiments. Proc. Natl. Acad. Sci. 2011, 108, 12734-12739.

(31) Kalinin, S.; Peulen, T.; Sindbert, S.; Rothwell, P. J.; Berger, S.; Restle, T.; Goody, R. S.; Gohlke, H.; Seidel, C. a M. A 
Toolkit and Benchmark Study for FRET-Restrained High-Precision Structural Modeling. Nat. Methods 2012, 9, 12181227.

(32) Stevens, J. A.; Link, J. J.; Zang, C.; Wang, L.; Zhong, D. Ultrafast Dynamics of Nonequilibrium Resonance Energy Transfer and Probing Globular Protein Flexibility of Myoglobin. J. Phys. Chem. A 2012, 116, 2610-2619.

(33) Milas, P.; Gamari, B. D.; Parrot, L.; Krueger, B. P.; Rahmanseresht, S.; Moore, J.; Goldner, L. S. Indocyanine Dyes Approach Free Rotation at the $3^{\prime}$ Terminus of A-RNA: A Comparison with the $5^{\prime}$ Terminus and Consequences for Fluorescence Resonance Energy Transfer. J. Phys. Chem. B 2013, 117, 8649-8658.

Hoefling, M.; Grubmüller, H. In Silico FRET from Simulated Dye Dynamics. Comput. Phys. Commun. 2013, 184, 841852.

(35) Kellner, R.; Hofmann, H.; Barducci, A.; Wunderlich, B.; Nettels, D.; Schuler, B. Single-Molecule Spectroscopy Reveals Chaperone-Mediated Expansion of Substrate Protein. Proc. Natl. Acad. Sci. 2014, 111, 13355-13360.

(36) Walczewska-Szewc, K.; Corry, B. Accounting for Dye Diffusion and Orientation When Relating FRET Measurements to Distances: Three Simple Computational Methods. Phys. Chem. Chem. Phys. 2014, 16, 12317-12326.

(37) Reif, M. M.; Oostenbrink, C. Molecular Dynamics Simulation of Configurational Ensembles Compatible with Experimental FRET Efficiency Data through a Restraint on Instantaneous FRET Efficiencies. J. Comput. Chem. 2014, 35, 2319-2332.

(38) Shoura, M. J.; Ranatunga, R. J. K. U.; Harris, S. a.; Nielsen, S. O.; Levene, S. D. Contribution of Fluorophore Dynamics and Solvation to Resonant Energy Transfer in Protein-DNA Complexes: A Molecular-Dynamics Study. Biophys. J. 2014, 107, 700-710.

(39) Søndergaard, S.; Aznauryan, M.; Haustrup, E. K.; Schiøtt, B.; Birkedal, V.; Corry, B. Dynamics of Fluorescent Dyes Attached to G-Quadruplex DNA and Their Effect on FRET Experiments. ChemPhysChem 2015, 16, 2562-2570.

(40) Kulesza, A.; Daly, S.; MacAleese, L.; Antoine, R.; Dugourd, P. Structural Exploration and Förster Theory Modeling for the Interpretation of Gas-Phase FRET Measurements: Chromophore-Grafted Amyloid- $\beta$ Peptides. J. Chem. Phys. 2015, 143, 25101.

(41) Jurinovich, S.; Curutchet, C.; Mennucci, B. The Fenna-Matthews-Olson Protein Revisited: A Fully Polarizable (TD)DFT/MM Description. ChemPhysChem 2014, 15, 3194-3204.

(42) Curutchet, C.; Mennucci, B. Quantum Chemical Studies of Light Harvesting. Chem. Rev. 2017, 117, 294-343.

(43) Curry, S. Lessons from the Crystallographic Analysis of Small Molecule Binding to Human Serum Albumin. Drug Metab. Pharmacokinet. 2009, 24, 342-357.

(44) Zhivkova, Z. Studies on Drug - Human Serum Albumin Binding: The Current State of the Matter. Curr. Pharm. Des. 2015, 21, 1817-1830.

(45) Davies, N. M. Clinical Pharmacokinetics of Flurbiprofen and Its Enantiomers. Clin. Pharmacokinet. 1995, 28, 100-114.

(46) Vayá, I.; Bueno, C. J.; Jiménez, M. C.; Miranda, M. A. Use of Triplet Excited States for the Study of Drug Binding to Human and Bovine Serum Albumins. ChemMedChem 2006, 1, 1015-1020.

(47) Lammers, I.; Lhiaubet-Vallet, V.; Consuelo Jiménez, M.; Ariese, F.; Miranda, M. A.; Gooijer, C. Stereoselective Binding of Flurbiprofen Enantiomers and Their Methyl Esters to Human Serum Albumin Studied by Time-Resolved Phosphorescence. Chirality 2012, 24, 840-846.

(48) Deschamps-Labat, L.; Péhourcq, F.; Jagou, M.; Bannwarth, B. Relationship between Lipophilicity and Binding to Human Serum Albumin of Arylpropionic Acid Non-Steroidal Anti-Inflammatory Drugs. J. Pharm. Biomed. Anal. 1997, 16, $223-$ 229.

(49) Østergaard, J.; Schou, C.; Larsen, C.; Heegaard, N. H. H. Effect of Dextran as a Run Buffer Additive in Drug-Protein Binding Studies Using Capillary Electrophoresis Frontal Analysis. Anal. Chem. 2003, 75, 207-214.

(50) Ghuman, J.; Zunszain, P. A.; Petitpas, I.; Bhattacharya, A. A.; Otagiri, M.; Curry, S. Structural Basis of the Drug-Binding Specificity of Human Serum Albumin. J. Mol. Biol. 2005, 353, 38-52.

(51) Curutchet, C.; Munoz-Losa, A.; Monti, S.; Kongsted, J.; Scholes, G. D.; Mennucci, B. Electronic Energy Transfer in Condensed Phase Studied by a Polarizable QM/MM Model. J. Chem. Theory Comput. 2009, 5, 1838-1848.

(52) Forster, T. Zwischenmolekulare Energiewanderung Und Fluoreszenz. Ann. Phys. 1948, 2, 55-75.

(53) Braslavsky, S. E.; Fron, E.; Rodríguez, H. B.; Román, E. S.; Scholes, G. D.; Schweitzer, G.; Valeur, B.; Wirz, J. Pitfalls and Limitations in the Practical Use of Förster's Theory of Resonance Energy Transfer. Photochem. Photobiol. Sci. 2008, $7,1444$. 
(55) Iozzi, M. F.; Mennucci, B.; Tomasi, J.; Cammi, R. Excitation Energy Transfer (EET) between Molecules in Condensed Matter: A Novel Application of the Polarizable Continuum Model (PCM). J. Chem. Phys. 2004, 120, 7029-7040.

(56) Curutchet, C.; Mennucci, B. Toward a Molecular Scale Interpretation of Excitation Energy Transfer in Solvated Bichromophoric Systems. J. Am. Chem. Soc. 2005, 127, 16733-16744.

(57) Case, D. A.; Darden, T. A.; T.E. Cheatham, I.; Simmerling, C. L.; Wang, J.; Duke, R. E.; Luo, R.; Walker, R. C.; Zhang, W.; Merz, K. M.; et al. AMBER 12. University of California: San Francisco, 2012.

(58) Olsson, M. H. M.; Søndergaard, C. R.; Rostkowski, M.; Jensen, J. H. PROPKA3: Consistent Treatment of Internal and Surface Residues in Empirical pKa Predictions. J. Chem. Theory Comput. 2011, 7, 525-537.

(59) Jorgensen, W. L.; Chandrasekhar, J.; Madura, J. D.; Impey, R. W.; Klein, M. L. Comparison of Simple Potential Functions for Simulating Liquid Water. J. Chem. Phys. 1983, 79, 926-935.

(60) Grabuleda, X.; Jaime, C.; Kollman, P. A. Molecular Dynamics Simulation Studies of Liquid Acetonitrile: New Six-Site Model. J. Comput. Chem. 2000, 21, 901-908.

(61) Wang, J.; Cieplak, P.; Kollman, P. A. How Well Does a Restrained Electrostatic Potential (RESP) Model Perform in Calculating Conformational Energies of Organic and Biological Molecules? J. Comput. Chem. 2000, 21, 1049-1074.

(62) Hornak, V.; Abel, R.; Okur, A.; Strockbine, B.; Roitberg, A.; Simmerling, C. Comparison of Multiple Amber Force Fields and Development of Improved Protein Backbone Parameters. Proteins-Structure Funct. Bioinforma. 2006, 65, 712-725.

(63) Grimme, S.; Antony, J.; Ehrlich, S.; Krieg, H. A Consistent and Accurate Ab Initio Parametrization of Density Functional Dispersion Correction (DFT-D) for the 94 Elements H-Pu. J. Chem. Phys. 2010, 132, 154104.

(64) Bayly, C. I.; Cieplak, P.; Cornell, W.; Kollman, P. A. A Well-Behaved Electrostatic Potential Based Method Using Charge Restraints for Deriving Atomic Charges: The RESP Model. J. Phys. Chem. 1993, 97, 10269-10280.

(65) Marenich, A. V.; Cramer, C. J.; Truhlar, D. G. Universal Solvation Model Based on Solute Electron Density and on a Continuum Model of the Solvent Defined by the Bulk Dielectric Constant and Atomic Surface Tensions. J. Phys. Chem. B 2009, 113, 6378-6396.

(66) Alecu, I. M.; Zheng, J.; Zhao, Y.; Truhlar, D. G. Computational Thermochemistry: Scale Factor Databases and Scale Factors for Vibrational Frequencies Obtained from Electronic Model Chemistries. J. Chem. Theory Comput. 2010, 6, $2872-2887$.

(67) Zerner, M. C. Semi Empirical Molecular Orbital Methods. In Reviews of Computational Chemistry, Vol. 2; Lipkowitz, K. B., Boyd, D. B., Eds.; VCH: New York, 1991; pp 313-366.

(68) Foresman, J. B.; Headgordon, M.; Pople, J. A.; Frisch, M. J. Toward a Systematic Molecular-Orbital Theory for ExcitedStates. J. Phys. Chem. 1992, 96, 135-149.

(69) Casida, M. E.; Huix-Rotllant, M. Progress in Time-Dependent Density-Functional Theory. Annu. Rev. Phys. Chem. 2012, $63,287-323$

(70) Yanai, T.; Tew, D. P.; Handy, N. C. A New Hybrid Exchange-correlation Functional Using the Coulomb-Attenuating Method (CAM-B3LYP). Chem. Phys. Lett. 2004, 393, 51-57.

(71) Frisch, M. J.; Trucks, G. W.; Schlegel, H. B.; Scuseria, G. E.; Robb, M. A.; Cheeseman, J. R.; Scalmani, G.; Barone, V.; Mennucci, B.; Petersson, G. A.; et al. Gaussian 09, Revision A.2. Gaussian, Inc.: Wallingford CT 2009.

(72) Wang, J.; Cieplak, P.; Li, J.; Hou, T.; Luo, R.; Duan, Y. Development of Polarizable Models for Molecular Mechanical Calculations I: Parameterization of Atomic Polarizability. J. Phys. Chem. B 2011, 115, 3091-3099.

(73) Wang, J.; Cieplak, P.; Li, J.; Wang, J.; Cai, Q.; Hsieh, M.; Lei, H.; Luo, R.; Duan, Y. Development of Polarizable Models for Molecular Mechanical Calculations II: Induced Dipole Models Significantly Improve Accuracy of Intermolecular Interaction Energies. J. Phys. Chem. B 2011, 115, 3100-3111.

(74) Wanko, M.; Hoffmann, M.; Strodel, P.; Koslowski, A.; Thiel, W.; Neese, F.; Frauenheim, T.; Elstner, M. Calculating Absorption Shifts for Retinal Proteins: Computational Challenges. J. Phys. Chem. B 2005, 109, 3606-3615.

(75) Aghtar, M.; Kleinekathöfer, U.; Curutchet, C.; Mennucci, B. Impact of Electronic Fluctuations and Their Description on the Exciton Dynamics in the Light-Harvesting Complex PE545. J. Phys. Chem. B 2017, 121, 1330-1339.

(76) Caricato, M.; Trucks, G. W.; Frisch, M. J.; Wiberg, K. B. Oscillator Strength: How Does TDDFT Compare to EOMCCSD? J. Chem. Theory Comput. 2011, 7, 456-466.

(77) Muñoz-Losa, A.; Curutchet, C.; Galván, I. F.; Mennucci, B. Quantum Mechanical Methods Applied to Excitation Energy Transfer: A Comparative Analysis on Excitation Energies and Electronic Couplings. J. Chem. Phys. 2008, $129,34104$.

(78) Knox, R. S. Dipole and Oscillator Strengths of Chromophores in Solution. Photochem. Photobiol. 2003, 77, 492-496.

(79) Knox, R. S.; Spring, B. Q. Dipole Strengths in the Chlorophylls. Photochem. Photobiol. 2003, 77, 497-501. 
TOC Graphic

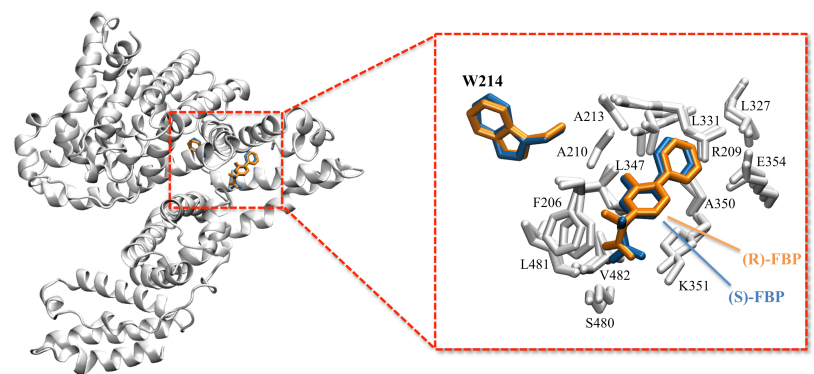

